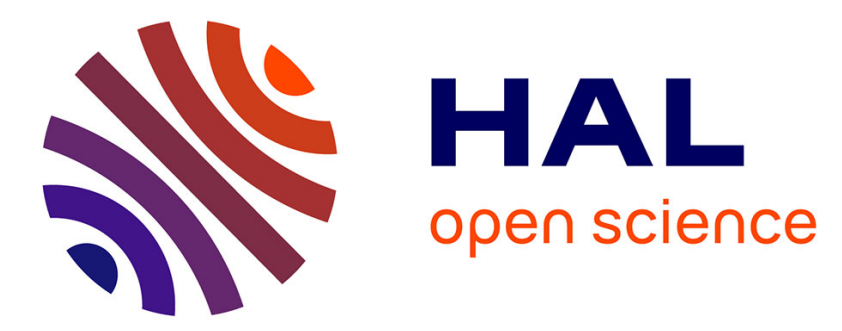

\title{
Effective pressure boundary condition for the filtration through porous medium via homogenization
}

\author{
Thomas Carraro, Eduard Marusic-Paloka, Andro Mikelic
}

\section{To cite this version:}

Thomas Carraro, Eduard Marusic-Paloka, Andro Mikelic. Effective pressure boundary condition for the filtration through porous medium via homogenization. Nonlinear Analysis: Real World Applications, 2018, 44, pp.149-172. 10.1016/j.nonrwa.2018.04.008 . hal-01484775

\section{HAL Id: hal-01484775 \\ https://hal.science/hal-01484775}

Submitted on 7 Mar 2017

HAL is a multi-disciplinary open access archive for the deposit and dissemination of scientific research documents, whether they are published or not. The documents may come from teaching and research institutions in France or abroad, or from public or private research centers.
L'archive ouverte pluridisciplinaire HAL, est destinée au dépôt et à la diffusion de documents scientifiques de niveau recherche, publiés ou non, émanant des établissements d'enseignement et de recherche français ou étrangers, des laboratoires publics ou privés. 


\title{
Effective pressure boundary condition for the filtration through porous medium via homogenization
}

\author{
Thomas Carraro ${ }^{1}$ \\ Institute for Applied Mathematics, Interdisciplinary Center for Scientific Computing \\ (IWR), Heidelberg University, Im Neuenheimer Feld 205, 69120 Heidelberg, Germany \\ Eduard Marušić-Paloka² \\ Department of mathematics, University of Zagreb, Bijenička 30, 10000 Zagreb, Croatia \\ Andro Mikelić ${ }^{3}$ \\ Univ Lyon, Université Claude Bernard Lyon 1, CNRS UMR 5208, \\ Institut Camille Jordan, 43 blvd. du 11 novembre 1918, \\ F-69622 Villeurbanne cedex, France
}

\begin{abstract}
We present homogenization of the viscous incompressible porous media flows under stress boundary conditions at the outer boundary. In addition to Darcy's law describing filtration in the interior of the porous medium, we derive rigorously the effective pressure boundary condition at the outer boundary. It is a linear combination of the outside pressure and the applied shear stress. We use the two-scale convergence in the sense of boundary layers, introduced by Allaire and Conca [SIAM J. Math. Anal., 29 (1997), pp. 343-379] to obtain the boundary layer structure next to the outer boundary. The approach allows establishing the strong $L^{2}$-convergence of the velocity corrector and identifica-

\footnotetext{
Email addresses: thomas.carraro@iwr.uni-heidelberg.de (Thomas Carraro), emarusic@math.hr (Eduard Marušić-Paloka), mikelic@univ-lyon1.fr (Andro Mikelić )

${ }^{1}$ The work of T.C. was supported by the German Research Council (DFG) through project "Multiscale modeling and numerical simulations of Lithium ion battery electrodes using real microstructures" (CA 633/2-1).

${ }^{2}$ The work of EMP was supported in part by the grant of the Croatian science foundation No 3955, Mathematical modelling and numerical simulations of processes in thin and porous domains

${ }^{3}$ The research of A.M. was supported in part by the LABEX MILYON (ANR-10-LABX0070) of Université de Lyon, within the program "Investissements d'Avenir" (ANR-11-IDEX0007) operated by the French National Research Agency (ANR).
} 
tion of the effective boundary velocity slip jump. The theoretical results are confirmed through numerical experiments.

Keywords: homogenization, stationary Navier-Stokes equations, stress boundary conditions, effective tangential velocity jump, porous media

\section{Introduction}

The porous media flows are of interest in a wide range of engineering disciplines including environmental and geological applications, flows through filters etc. They take place in a material which consists of a solid skeleton and bil5 lions of interconnected fluid filled pores. The flows are characterised by large spatial and temporal scales. The complex geometry makes direct computing of the flows, and also reactions, deformations and other phenomena, practically impossible. In the applications, the mesoscopic modeling is privileged and one search for effective models where the information on the geometry is kept in the coefficients and which are valid everywhere. The technique which allows replacing the physical models posed at the microstructure level by equations valid globally, is called upscaling. Its mathematical variant, which gives also the rigorous relationship between the upscaled and the microscopic models is the homogenization technique.

It has been applied to a number of porous media problems, starting from the seminal work of Tartar 28 and the monograph 27. Many subjects are reviewed in the book [16. See also the references therein.

Frequently, one has processes on multiple domains and model-coupling approaches are needed. Absence of the statistical homogeneity does not allow direct use of the homogenization techniques. Examples of situations where the presence of an interface breaks the statistical homogeneity are

- the flow of a viscous fluid over a porous bed,

- the forced infiltration into a porous medium. 
The tangential flow of an unconfined fluid over a porous bed is described by the law of Beavers and Joseph [5] and it was rigorously derived in [19] and [23] using a combination of the homogenization and boundary layer techniques. The forced injection problem was introduced in [21] and the interface conditions were rigorously established and justified in [10].

A particular class of the above problems is derivation of the homogenized external boundary conditions for the porous media flows. In the case of the zero velocity at the external boundary of the porous medium, one would impose zero normal component of the Darcy velocity as the homogenized boundary condition. The behavior of the velocity and pressure field close to the flat external boundary, with such boundary condition, has been studied in [17, using 35 the technique from [18. The error estimate in $2 \mathrm{D}$, for an arbitrary geometry has been established in [24].

The case of the velocity boundary conditions could be considered as "intuitively" obvious. Other class of problems arises when we have a contact of the porous medium with another fluid flow and the normal contact force is given at the boundary. It describes the physical situation when the upper boundary of the porous medium in exposed to the atmospheric pressure and wind (see e.g. [12]). Or, more generally, when the fluid that we study is in contact with another given fluid. Assuming that the motion in porous medium is slow enough that the interface $\Sigma$ between two fluids can be seen as immobile. Intuitively, it

45 is expected that the homogenized pressure will take the prescribed value at the boundary.

In this article we study the homogenization of the stationary Navier-Stokes equations with the given normal contact force at the external boundary and we will find out that the result is more rich than expected.

\section{Setting of the problem}

We start by defining the geometry. Let $\ell$ and $d$ be two positive constants. Let $\Omega=(0, \ell) \times(-d, 0) \subset \mathbb{R}^{2}$ be a rectangle. We denote the upper boundary 
by

$$
\Sigma=\left\{\left(x_{1}, 0\right) \in \mathbb{R}^{2} ; x_{1} \in(0, \ell)\right\} .
$$

The bottom of the domain is denoted by

$$
\Gamma=\left\{\left(x_{1},-d\right) \in \mathbb{R}^{2} ; x_{1} \in(0, \ell)\right\}
$$

We set $\Gamma=\partial \Omega \backslash \Sigma$. Let $A \subset \subset \mathbb{R}^{2}$ be a smooth domain such that $\bar{A} \subset$ $(0,1)^{2} \equiv Y$. The unit pore is $Y^{*}=Y \backslash \bar{A}$. Now we choose the small parameter $\varepsilon \ll 1$ such that $\varepsilon=\ell / m$, with $m \in \mathbb{N}$ and define

$$
T_{\varepsilon}=\left\{\mathbf{k} \in \mathbb{Z}^{2} ; \varepsilon(\mathbf{k}+A) \subset \Omega\right\}, Y_{\varepsilon, k}^{*}=\varepsilon\left(\mathbf{k}+Y^{*}\right), A_{k}^{\varepsilon}=\varepsilon(\mathbf{k}+A) .
$$

The fluid part of the porous medium is now $\Omega_{\varepsilon}=\Omega \backslash \overline{\bigcup_{\mathbf{k} \in T_{\varepsilon}} \varepsilon(\mathbf{k}+A)}$. Finally, $B_{\varepsilon}=\overline{\bigcup_{\mathbf{k} \in T_{\varepsilon}} \varepsilon(\mathbf{k}+A)}$ is the solid part of the porous medium and its boundary is $S_{\varepsilon}=\partial B_{\varepsilon}$.

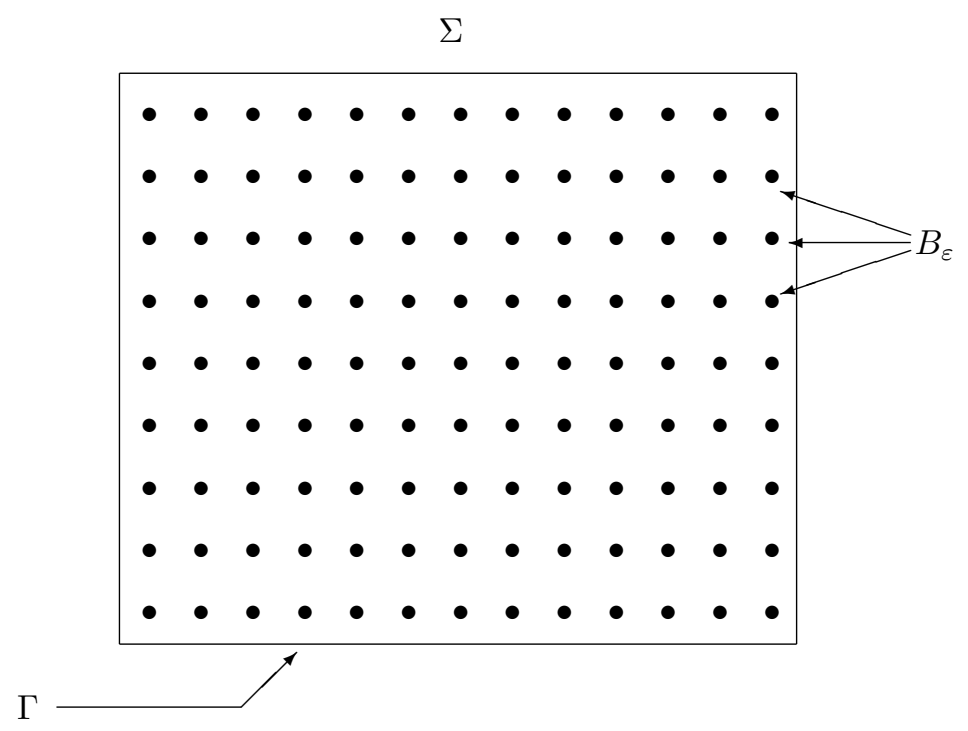


On $\Sigma$ we prescribe the normal stress and $\Gamma$ is an impermeable boundary. In the dimensionless form, the Stokes problem that we study reads

$$
\begin{aligned}
& -\mu \Delta \mathbf{u}^{\varepsilon}+\nabla p^{\varepsilon}=\mathbf{F}, \operatorname{div} \mathbf{u}^{\varepsilon}=0 \text { in } \Omega_{\varepsilon}, \\
& \mathbf{T}\left(\mathbf{u}^{\varepsilon}, p^{\varepsilon}\right) \mathbf{e}_{2}=\mathbf{H}=(P, Q) \text { on } \Sigma, \quad \mathbf{u}^{\varepsilon}=0 \text { on } S_{\varepsilon} \cup \Gamma, \\
& \left(\mathbf{u}^{\varepsilon}, p^{\varepsilon}\right) \text { is } \ell \text {-periodic in } x_{1} .
\end{aligned}
$$

Here $\mathbf{T}(\mathbf{v}, q)$ denotes the stress tensor and $\mathbf{D} \mathbf{v}$ the rate of strain tensor

$$
\mathbf{T}(\mathbf{v}, q)=-2 \mu \mathbf{D} \mathbf{v}+q \mathbf{I}, \mathbf{D v}=\frac{1}{2}\left(\nabla \mathbf{v}+(\nabla \mathbf{v})^{t}\right)
$$

and $\mu$ is a positive constant.

Assumption 1. We suppose $\partial A \in C^{3}, \mathbf{F} \in C^{1}(\bar{\Omega})^{2}$ and $P=P\left(x_{1}\right), Q=Q\left(x_{1}\right)$ being elements of $C_{\text {per }}^{1}[0, \ell]$.

75 For the existence, uniqueness and regularity of solutions to Stokes problem (1)(3), under Assumption 1, we refer e.g. to [7, Sec. 4.7.

Furthermore, we consider the full stationary incompressible Navier-Stokes system

$$
\begin{aligned}
& -\mu \Delta \mathbf{u}^{1, \varepsilon}+\left(\mathbf{u}^{1, \varepsilon} \nabla\right) \mathbf{u}^{1, \varepsilon}+\nabla p^{1, \varepsilon}=\mathbf{F}, \operatorname{div} \mathbf{u}^{1, \varepsilon}=0 \text { in } \Omega_{\varepsilon} \\
& \mathbf{T}\left(\mathbf{u}^{1, \varepsilon}, p^{1, \varepsilon}\right) \mathbf{e}_{2}=\mathbf{H}=(P, Q) \text { on } \Sigma, \quad \mathbf{u}^{1, \varepsilon}=0 \text { on } S_{\varepsilon} \cup \Gamma \\
& \left(\mathbf{u}^{1, \varepsilon}, p^{1, \varepsilon}\right) \text { is } \ell \text {-periodic in } x_{1} .
\end{aligned}
$$

Existence of a solution for problem (4)-(6) is discussed in Sec. 5.

so Our goal is to study behavior of solutions to (1)-(3) and (4)-(6) in the limit when the small parameter $\varepsilon \rightarrow 0$.

\section{The main result}

Our goal is to describe the effective behavior of the fluid flow in the above described situation. The filtration in the bulk is expected to be described by

${ }_{85}$ Darcy's law and we are looking for the effective boundary condition on the upper 
boundary $\Sigma$. To do so, we apply various homogenization techniques, such as two-scale convergence $([26],[1)$ and the two-scale convergence for boundary layers ([3]). We prove the following result:

Theorem 1. Let us suppose Assumption 1 and let $\left(\mathbf{u}^{\varepsilon}, p^{\varepsilon}\right)$ be the solution of 90 problem (1)- (3).

Then there exists an extension of $p^{\varepsilon}$ to the whole $\Omega$, denoted again by the same symbol, such that

$$
p^{\varepsilon} \rightarrow p^{0} \quad \text { strongly in } L^{2}(\Omega) \text {, }
$$

where $p^{0}$ is the solution of problem

$$
\begin{aligned}
& \operatorname{div} \mathbf{K}\left(\nabla p^{0}-\mathbf{F}\right)=0 \text { in } \Omega, \\
& p^{0} \text { is } \ell \text {-periodic in } x_{1}, \quad \mathbf{n} \cdot \mathbf{K}\left(\nabla p^{0}-\mathbf{F}\right)=0 \text { on } \Gamma, \\
& p^{0}=C_{\pi} P+Q \text { on } \Sigma,
\end{aligned}
$$

with $\mathbf{K}$ the permeability tensor, defined by 83), and $C_{\pi}$ the boundary layer pressure stabilisation constant given by (41).

Next, let $(\mathbf{w}, \pi)$ be the solution of the boundary layer problem (36)-(38).

Then, after extending $\mathbf{u}^{\varepsilon}$ and $\mathbf{w}$ by zero to the perforations, we have

$$
\begin{gathered}
\quad \frac{\mathbf{u}^{\varepsilon}(x)-\varepsilon P\left(x_{1}\right) \mathbf{w}(x / \varepsilon)}{\varepsilon^{2}} \rightarrow \mathbf{V} \text { weakly in } L^{2}(\Omega) \\
\frac{\mathbf{u}^{\varepsilon}(x)}{\varepsilon^{2}} \rightarrow P\left(x_{1}\right)\left(\int_{G^{*}} w_{1}(y) d y\right) \delta_{\Sigma} \mathbf{e}_{1}+\mathbf{V} \text { weak } \text { in }^{*} \mathcal{M}(\Omega) \\
\frac{\mathbf{u}^{\varepsilon}-\varepsilon P\left(x_{1}\right) \mathbf{w}\left(\frac{x}{\varepsilon}\right)}{\varepsilon^{2}}-\sum_{k=1}^{2} \mathbf{w}^{k}\left(\frac{x}{\varepsilon}\right)\left(F_{k}-\frac{\partial p^{0}}{\partial x_{k}}\right) \rightarrow 0 \text { strongly in } L^{2}(\Omega),(13)
\end{gathered}
$$

where $G^{*}$ is the boundary layer internal interface fluid/solid given by (28), $\mathbf{V}$ satisfies the Darcy law

$$
\mathbf{V}=\mathbf{K}\left(\mathbf{F}-\nabla p^{0}\right)
$$

$\mathcal{M}(\Omega)$ denotes the set of Radon measures on $\Omega$ and $\delta_{\Sigma}$ is the Dirac measure concentrated on $\Sigma$, i.e. $\left\langle\delta_{\Sigma} \mid \psi\right\rangle=\int_{\Sigma} \psi\left(x_{1}, 0\right) d x_{1}$.

An analogous result holds for the homogenization of the stationary NavierStokes equations (4)-(6) 
Theorem 2. Under the assumptions on the geometry and the data from Theorem (1), there exist solutions $\left(\mathbf{u}^{1, \varepsilon}, p^{1, \varepsilon}\right)$ of problem (4)- (6) such that convergences (7), (11)-13) take place.

\section{Proof of Theorem 1}

The proof is divided in several steps. First we derive the a priori estimates. Then we pass to the two-scale limit for boundary layers, in order to determine the local behavior of the solution in vicinity of the boundary. Once it is achieved, we subtract the boundary layer corrector from the original solution and use the classical two-scale convergence to prove that the residual converges towards the limit that satisfies the Darcy law. At the end we prove the strong convergences.

\subsection{Step one: A priori estimates}

We first recall that in $\Omega_{\varepsilon}$ Poincaré and trace constants depend on $\varepsilon$ in the following way

$$
\begin{aligned}
& |\phi|_{L^{2}\left(\Omega_{\varepsilon}\right)} \leq C \varepsilon|\nabla \phi|_{L^{2}\left(\Omega_{\varepsilon}\right)} \\
& |\phi|_{L^{2}(\Sigma)} \leq C \sqrt{\varepsilon}|\nabla \phi|_{L^{2}\left(\Omega_{\varepsilon}\right)}, \forall \phi \in H^{1}\left(\Omega_{\varepsilon}\right), \phi=0 \text { on } S_{\varepsilon}
\end{aligned}
$$

We also recall that the norms $|\mathbf{D v}|_{L^{2}\left(\Omega_{\varepsilon}\right)}$ and $|\nabla \mathbf{v}|_{L^{2}\left(\Omega_{\varepsilon}\right)}$ are equivalent, due to the Korn's inequality, which is independent of $\varepsilon$ (see e.g. [7]).

Here and in the sequel we assume that $\mathbf{u}^{\varepsilon}$ is extended by zero to the whole $\Omega$. In order to extend the pressure $p^{\varepsilon}$ we need Tartar's construction from his seminal paper [28]. It relies on the related construction of the restriction operator, acting from the whole domain $\Omega$ to the pore space $\Omega_{\varepsilon}$. In our setting we deal with the functional spaces

$$
\begin{aligned}
& X_{2}=\left\{\mathbf{z} \in H^{1}(\Omega)^{2} ; \mathbf{z}=0 \text { for } x_{2}=-d\right\} \\
& X_{2}^{\varepsilon}=\left\{\mathbf{z} \in X_{2} ; \mathbf{z}=0 \text { on } S_{\varepsilon}\right\} .
\end{aligned}
$$


Then, after 28] and the detailed review in [2], there exists a continuous restriction operator $R_{\varepsilon} \in \mathcal{L}\left(X_{2}, X_{2}^{\varepsilon}\right)$, such that

$$
\begin{aligned}
& \operatorname{div}\left(R_{\varepsilon} \mathbf{z}\right)=\operatorname{div} \mathbf{z}+\sum_{\mathbf{k} \in T_{\varepsilon}} \frac{1}{\left|Y_{\varepsilon, k}^{*}\right|} \chi_{\varepsilon, k} \int_{A_{k}^{\varepsilon}} \operatorname{div} \mathbf{z} d x, \quad \forall \mathbf{z} \in X_{2}, \\
& \left|R_{\varepsilon} \mathbf{z}\right|_{L^{2}\left(\Omega_{\varepsilon}\right)} \leq C\left(\varepsilon|\nabla \mathbf{z}|_{L^{2}(\Omega)}+|\mathbf{z}|_{L^{2}(\Omega)}\right), \forall \mathbf{z} \in X_{2}, \\
& \left|\nabla R_{\varepsilon} \mathbf{z}\right|_{L^{2}\left(\Omega_{\varepsilon}\right)} \leq \frac{C}{\varepsilon}\left(\varepsilon|\nabla \mathbf{z}|_{L^{2}(\Omega)}+|\mathbf{z}|_{L^{2}(\Omega)}\right), \forall \mathbf{z} \in X_{2},
\end{aligned}
$$

where $\chi_{\varepsilon, k}$ denotes the characteristic function of the set $Y_{\varepsilon, k}^{*}, \mathbf{k} \in T_{\varepsilon}$. Through a duality argument, it gives an extension of the pressure gradient and it was found in 22] that the pressure extension $\tilde{p}$ is given by the explicit formula

$$
\tilde{p}^{\varepsilon}=\left\{\begin{array}{l}
p^{\varepsilon} \text { in } \Omega_{\varepsilon} \\
\frac{1}{\left|Y_{\varepsilon, k}^{*}\right|} \int_{Y_{\varepsilon, k}^{*}} p^{\varepsilon} d x \text { in } Y_{\varepsilon, k}^{*} \text { for each } \mathbf{k} \in T_{\varepsilon} .
\end{array}\right.
$$

For details we refer to [2]. In addition, a direct computation yields

$$
\int_{\Omega_{\varepsilon}} p^{\varepsilon} \operatorname{div}\left(R_{\varepsilon} \mathbf{z}\right) d x=\int_{\Omega} \tilde{p}^{\varepsilon} d x \operatorname{div} \mathbf{z} d x, \forall \mathbf{z} \in X_{2} .
$$

Both the velocity and the pressure extensions are, for simplicity, denoted by the same symbols as the original functions $\left(\mathbf{u}^{\varepsilon}, p^{\varepsilon}\right)$.

It is straightforward to see that:

Lemma 1. Let $\left(\mathbf{u}^{\varepsilon}, p^{\varepsilon}\right)$ be the solution to problem (1), (2). Then there exists some constant $C>0$, independent of $\varepsilon$, such that

$$
\begin{aligned}
& \left|\nabla \mathbf{u}^{\varepsilon}\right|_{L^{2}(\Omega)} \leq C \sqrt{\varepsilon} \\
& \left|\mathbf{u}^{\varepsilon}\right|_{L^{2}(\Omega)} \leq C \varepsilon^{3 / 2} \\
& \left|p^{\varepsilon}\right|_{L^{2}(\Omega)} \leq \frac{C}{\sqrt{\varepsilon}} .
\end{aligned}
$$

Proof. We start from the variational formulation of problem (1), (2)

$$
\begin{gathered}
\mu \int_{\Omega_{\varepsilon}} \mathbf{D u}^{\varepsilon}: \mathbf{D} \mathbf{v} d x=\int_{\Sigma} \mathbf{H} \cdot \mathbf{v} d S+\int_{\Omega_{\varepsilon}} \mathbf{F} \cdot \mathbf{v} d x, \forall \mathbf{v} \in V\left(\Omega_{\varepsilon}\right), \\
V\left(\Omega_{\varepsilon}\right)=\left\{\mathbf{v} \in H^{1}\left(\Omega_{\varepsilon}\right)^{2} ; \operatorname{div} \mathbf{v}=0, \mathbf{v}=0 \text { on } S_{\varepsilon} \cup \Gamma, \mathbf{v} \text { is } \ell-\text { periodic in } x_{1}\right\}
\end{gathered}
$$


Using $\mathbf{u}^{\varepsilon}$ as the test function and applying (14)-(15) yield

$$
\mu \int_{\Omega_{\varepsilon}}\left|\mathbf{D} \mathbf{u}^{\varepsilon}\right|^{2} d x=\int_{\Sigma} \mathbf{H} \cdot \mathbf{u}^{\varepsilon} d S+\int_{\Omega_{\varepsilon}} \mathbf{F} \cdot \mathbf{u}^{\varepsilon} d x \leq C \sqrt{\varepsilon}\left|\mathbf{D} \mathbf{u}^{\varepsilon}\right|_{L^{2}\left(\Omega_{\varepsilon}\right)} .
$$

Now 14 implies 18 and 19 . Since we have extended the pressure to the solid part of $\Omega$, using Tartar's construction, (18) and (17) imply

$$
\left|p^{\varepsilon}\right|_{L^{2}(\Omega) / \mathbb{R}}=\sup _{g \in L^{2}(\Omega) / \mathbb{R}} \frac{\int_{\Omega} p^{\varepsilon} g d x}{|g|_{L^{2}(\Omega) / \mathbb{R}}}=\sup _{\mathbf{z} \in X_{2}} \frac{\int_{\Omega_{\varepsilon}} p^{\varepsilon} \operatorname{div}\left(\mathbf{R}_{\varepsilon} \mathbf{z}\right) d x}{|\mathbf{z}|_{H^{1}(\Omega)^{2}}} \leq \frac{C}{\varepsilon}\left|\nabla \mathbf{u}^{\varepsilon}\right|_{L^{2}(\Omega)},
$$

giving the pressure estimate 20 .

\subsection{Step two: Two-scale convergence for boundary layers}

We recall the definition and some basic compactness results for two-scale convergence for boundary layers due to Allaire and Conca [3]. In the sequel, if the index $y$ is added to the differential operators $\mathbf{D}_{y}, \nabla_{y}, \operatorname{div}_{y}$, then the derivatives are taken with respect to the fast variables $y_{1}, y_{2}$ instead of $x_{1}, x_{2}$. Let $G=(0,1) \times(-\infty, 0)$ be an infinite band. The bounded sequence $\left(\phi_{\varepsilon}\right)_{\varepsilon>0} \subset$ $L^{2}(\Omega)$ is said to two-scale converge in the sense of the boundary layers if there exists $\phi_{0}\left(x_{1}, y\right) \in L^{2}(\Sigma \times G)$ such that

$$
\frac{1}{\varepsilon} \int_{\Omega} \phi_{\varepsilon}(x) \psi\left(x_{1}, \frac{x}{\varepsilon}\right) d x \rightarrow \int_{\Sigma} \int_{G} \phi_{0}\left(x_{1}, y\right) \psi\left(x_{1}, y\right) d x_{1} d y,
$$

for all smooth functions $\psi\left(x_{1}, y\right)$ defined in $\Sigma \times \bar{G}$, with bounded support, such that $y_{1} \mapsto \psi\left(x_{1}, y_{1}, y_{2}\right)$ is 1-periodic.

We need the following functional space

$$
\begin{aligned}
& \mathcal{D}^{1}=\left\{\psi \in C^{\infty}(G) ; \psi \text { is } 1-\text { periodic in } y_{1}\right. \\
& \text { and compactly supported in } \left.y_{2} \in(-\infty, 0]\right\}
\end{aligned}
$$

Now $D_{\#}^{1}(G)$ is the closure of $\mathcal{D}^{1}$ in the norm $|\psi|_{D_{\#}^{1}(G)}=|\nabla \psi|_{L^{2}(G)}$. It should

${ }_{130}$ be noticed that such functions do not necessarily vanish as $y_{2} \rightarrow-\infty$. For that kind of convergence we have the following compactness result from [3]:

Theorem 3. 1. Let us suppose

$$
\frac{1}{\sqrt{\varepsilon}}\left|\phi_{\varepsilon}\right|_{L^{2}(\Omega)} \leq C
$$


Then there exists $\phi_{0} \in L^{2}(\Sigma \times G)$ and a subsequence, denoted by the same indices, such that

$$
\phi_{\varepsilon} \rightarrow \phi_{0} \text { two-scale in the sense of boundary layers. }
$$

2. Let us suppose

$$
\frac{1}{\sqrt{\varepsilon}}\left(\left|\phi_{\varepsilon}\right|_{L^{2}(\Omega)}+\varepsilon\left|\nabla \phi_{\varepsilon}\right|_{L^{2}(\Omega)}\right) \leq C .
$$

Then there exists $\phi_{0} \in L^{2}\left(\Sigma ; D_{\#}^{1}(G)\right)$ and a subsequence, denoted by the same indices, such that

$$
\begin{aligned}
& \phi_{\varepsilon} \rightarrow \phi_{0} \text { two-scale in the sense of boundary layers } \\
& \varepsilon \nabla \phi_{\varepsilon} \rightarrow \nabla_{y} \phi_{0} \text { two-scale in the sense of boundary layers }
\end{aligned}
$$

Using the a priori estimates, we now undertake our first passing to the limit.

Before we start we define

$$
\mathcal{C}=\bigcup_{j=0}^{-\infty}\left(j \mathbf{e}_{2}+\partial A\right), M=\bigcup_{j=0}^{-\infty}\left(j \mathbf{e}_{2}+\bar{A}\right), G^{*}=G \backslash \bigcup_{j=0}^{-\infty}\left(j \mathbf{e}_{2}+A\right)
$$

We introduce the space $D_{\# 0}^{1}\left(G^{*}\right)$ defined similarly as $D_{\#}^{1}(G)$ but on $G^{*}$ and such that its elements have zero trace on $\mathcal{C}$. Thus, we take

$$
\begin{aligned}
& \mathcal{D}^{1}=\left\{\psi \in C^{\infty}\left(G^{*}\right) ;\left.\psi\right|_{\mathcal{C}}=0, \psi \text { is } 1-\text { periodic in } y_{1}\right. \\
&\text { and compactly supported in } \left.y_{2} \in(-\infty, 0]\right\}
\end{aligned}
$$

Then $D_{\# 0}^{1}\left(G^{*}\right)$ is its closure in the norm $|\psi|_{D_{\# 0}^{1}\left(G^{*}\right)}=|\nabla \psi|_{L^{2}\left(G^{*}\right)}$. Those functions do vanish as $y_{2} \rightarrow-\infty$ due to the zero trace on $\mathcal{C}$ that prevents them to tend to a constant.

Lemma 2. Let $\left(\mathbf{v}^{0}, q^{0}\right) \in L^{2}\left(\Sigma ; D_{\# 0}^{1}\left(G^{*}\right)\right) \times L^{2}\left(\Sigma ; L_{l o c}^{2}\left(G^{*}\right)\right)$ be given by the boundary layer problem

$$
\begin{aligned}
& -\mu \Delta_{y} \mathbf{v}^{0}+\nabla_{y} q^{0}=0, \quad d i v_{y} \mathbf{v}^{0}=0 \text { in } G^{*} \\
& \left(-2 \mu \mathbf{D}_{y} \mathbf{v}^{0}+q^{0} \mathbf{I}\right) \mathbf{e}_{2}=\mathbf{H} \text { for } y_{2}=0, \mathbf{v}^{0}=0 \text { on } \mathcal{C} \\
& \left(\mathbf{v}^{0}, q^{0}\right) \text { is 1-periodic in } y_{1}, \mathbf{v}^{0} \rightarrow 0 \text { as } y_{2} \rightarrow-\infty
\end{aligned}
$$


Then

$$
\begin{aligned}
& \frac{1}{\varepsilon} \mathbf{u}^{\varepsilon} \rightarrow \mathbf{v}^{0} \text { two-scale in the sense of boundary layers } \\
& \nabla \mathbf{u}^{\varepsilon} \rightarrow \nabla_{y} \mathbf{v}^{0} \text { two-scale in the sense of boundary layers. }
\end{aligned}
$$

140 Proof. The a priori estimates $(19)$ and $(18)$ and the compactness theorem 3 imply the existence of some $\mathbf{v}^{0} \in L^{2}\left(\Sigma ; D_{\# 0}^{1}\left(G^{*}\right)\right)$ such that $\mathbf{v}^{0}=0$ on $M$ and

$$
\begin{aligned}
& \frac{1}{\varepsilon} \mathbf{u}^{\varepsilon} \rightarrow \mathbf{v}^{0} \text { two-scale in the sense of boundary layers } \\
& \nabla \mathbf{u}^{\varepsilon} \rightarrow \nabla_{y} \mathbf{v}^{0} \text { two-scale in the sense of boundary layers . }
\end{aligned}
$$

Now we take the test function $\mathbf{z}^{\varepsilon}(x)=\mathbf{z}\left(x_{1}, \frac{x}{\varepsilon}\right) \in D_{\# 0}^{1}\left(G^{*}\right)^{2}$ such that $\operatorname{div}_{y} \mathbf{z}=0$ and $\mathbf{z}\left(x_{1}, \cdot\right)=0$ in $M$ in the variational formulation for (1), (2)

$$
\frac{2 \mu}{\varepsilon} \int_{\Omega_{\varepsilon}} \varepsilon \mathbf{D} \frac{\mathbf{u}^{\varepsilon}}{\varepsilon}: \varepsilon \mathbf{D} \mathbf{z}^{\varepsilon} d x-\int_{\Omega_{\varepsilon}} p^{\varepsilon} \operatorname{div} \mathbf{z}^{\varepsilon} d x=\int_{\Sigma} \mathbf{H} \cdot \mathbf{z}^{\varepsilon} d S+\int_{\Omega_{\varepsilon}} \mathbf{F} \cdot \mathbf{z}^{\varepsilon} d x
$$

Since

$$
\frac{\partial \mathbf{z}^{\varepsilon}}{\partial x_{j}}=\varepsilon^{-1} \frac{\partial \mathbf{z}}{\partial y_{j}}+\delta_{1 j} \frac{\partial \mathbf{z}}{\partial x_{1}}
$$

we get on the limit

$2 \mu \int_{\Sigma}\left(\int_{G} \mathbf{D}_{y} \mathbf{v}^{0}\left(x_{1}, y\right): \mathbf{D}_{y} \mathbf{z}\left(x_{1}, y\right) d y\right) d x_{1}=\int_{\Sigma} \mathbf{H} \cdot\left(\int_{0}^{1} \mathbf{z}\left(x_{1}, y_{1}, 0\right) d y_{1}\right) d x_{1}$.

Furthermore, since $\operatorname{div} \mathbf{u}^{\varepsilon}=0$ it easily follows that $\operatorname{div}_{y} \mathbf{v}^{0}=0$. Thus there exists $q^{0} \in L^{2}\left(\Sigma ; L_{l o c}^{2}\left(G^{*}\right)\right)$ such that $\left(\mathbf{v}^{0}, q^{0}\right)$ satisfy $29-31$.

145 The boundary layer corrector $\left(\mathbf{v}^{0}, q^{0}\right)$ can be decomposed as $\mathbf{v}^{0}=P\left(x_{1}\right) \mathbf{w}(y)$ and $q^{0}=P\left(x_{1}\right) \pi(y)+Q\left(x_{1}\right)$, where

$$
\begin{aligned}
& -\mu \Delta_{y} \mathbf{w}+\nabla_{y} \pi=0, \operatorname{div}_{y} \mathbf{w}=0 \text { in } G^{*}, \\
& \left(-2 \mu \mathbf{D}_{y} \mathbf{w}+\pi \mathbf{I}\right) \mathbf{e}_{2}=\mathbf{e}_{1} \text { for } y_{2}=0, \mathbf{w}=0 \text { on } \mathcal{C}, \\
& (\mathbf{w}, \pi) \text { is 1-periodic in } y_{1}, \mathbf{w} \rightarrow 0 \text { as } y_{2} \rightarrow-\infty .
\end{aligned}
$$

Problem (36), (38) is of the boundary layer type. Existence of the solution and exponential decay can be proved as in [18]. We have 
Theorem 4. Problem (36), (38) has a unique solution $(\mathbf{w}, \pi) \in D_{\# 0}^{1}\left(G^{*}\right) \times$ $L_{\text {loc }}^{2}\left(G^{*}\right)$. Furthermore, there exists a constant $C_{\pi}$ such that

$$
\begin{aligned}
& \left|e^{\alpha\left|y_{2}\right|}\left(\pi-C_{\pi}\right)\right|_{L^{2}\left(G^{*}\right)} \leq C \\
& \left|e^{\alpha\left|y_{2}\right|} \mathbf{w}\right|_{L^{2}\left(G^{*}\right)}+\left|e^{\alpha\left|y_{2}\right|} \nabla \mathbf{w}\right|_{L^{2}\left(G^{*}\right)} \leq C .
\end{aligned}
$$

for some constants $C, \alpha>0$.

In the sense of 39 we write

$$
C_{\pi}=\lim _{y_{2} \rightarrow-\infty} \pi(y)
$$

Remark 1. If $\partial A \in C^{3}$ then the regularity theory for the Stokes operator applies and (39), (40) hold pointwise. For more details on the regularity see e.g.

Remark 2. Let the solution $\mathbf{w}$ to system (36)- (38) be extended by zero to M. Let $b>a>0$ be arbitrary constants. Then we have

$$
0=\int_{a}^{b} \int_{0}^{1} \operatorname{div} \mathbf{w} d y=\int_{0}^{1} w_{2}\left(y_{1}, b\right) d y_{1}-\int_{0}^{1} w_{2}\left(y_{1}, a\right) d y_{1} .
$$

Using (40) yields

$$
\int_{0}^{1} w_{2}\left(y_{1}, y_{2}\right) d y_{1}=0, \quad \forall y_{2} \leq 0
$$

(42) implies

$$
\int_{G^{*}} w_{2} d y=0
$$

Remark 3. Integrating (37) with respect to $y_{1}$ yields

$$
\mathbf{e}_{1}=\int_{0}^{1}\left[-\mu\left(\frac{\partial w_{1}}{\partial y_{2}} \mathbf{e}_{1}+\frac{\partial w_{2}}{\partial y_{1}} \mathbf{e}_{1}+2 \frac{\partial w_{2}}{\partial y_{2}} \mathbf{e}_{2}\right)+\pi \mathbf{e}_{2}\right]\left(y_{1}, 0\right) d y_{1} .
$$

Equating the second components gives

$$
\begin{aligned}
0 & =\int_{0}^{1}\left(-2 \mu \frac{\partial w_{2}}{\partial y_{2}}+\pi\right)\left(y_{1}, 0\right) d y_{1}=\int_{0}^{1}\left(2 \mu \frac{\partial w_{1}}{\partial y_{1}}+\pi\right)\left(y_{1}, 0\right) d y_{1}= \\
& =\int_{0}^{1} \pi\left(y_{1}, 0\right) d y_{1} .
\end{aligned}
$$


If we test (36) with $\mathbf{w}^{k}$ and (80) by $\mathbf{w}$ and combine, we get

$C_{\pi}=K_{22}^{-1}\left(\int_{0}^{1} w_{1}^{2}\left(y_{1}, 0\right) d y_{1}+\int_{0}^{1}\left(-2 \mu \frac{\partial \mathbf{w}^{k}}{\partial y_{2}}+\pi^{k} \mathbf{e}_{2}\right)\left(y_{1}, 0\right) \mathbf{w}\left(y_{1}, 0\right) d y_{1}\right)$.

Finally, we denote $\left.\left.J=\left\{y_{2} \in\right]-\infty, 0\right] ;\left(y_{1}, y_{2}\right) \notin M, y_{1} \in\right] 0,1[\}$. Denoting

$m_{A}=\min \left\{y_{2} \in[0,1] ;\left(y_{1}, y_{2}\right) \in \bar{A}\right\}, M_{A}=\max \left\{y_{2} \in[0,1]:\left(y_{1}, y_{2}\right) \in \bar{A}\right\}$

The set $J$ is then a union of disjoint intervals $\left.J_{0}=\right] 0, m_{A}\left[, J_{i}=\right] i-1+$ $M_{A}, i+m_{a}\left[, i=1,2, \ldots\right.$ It is easy to see that the mapping $t \mapsto \int_{0}^{1} \pi\left(y_{1}, t\right) d y_{1}$ is constant on each of the intervals $J_{i}$. If those constants are denoted by $c_{i}$ then $c_{0}=0$ and $\lim _{i \rightarrow \infty} c_{i}=C_{\pi}$.

Remark 4. Let us suppose that the boundary layer geometry has the mirror symmetry with respect to the axis $\left\{y_{1}=1 / 2\right\}$. Then $w_{2}$ and $\pi$ are uneven functions with respect to the axis and $C_{\pi}=0$. In particular, this result applies to the case of circular inclusions.

We now know the behavior of $\left(\mathbf{u}^{\varepsilon}, p^{\varepsilon}\right)$ in vicinity of $\Sigma$. To get additional information of the behavior far from the boundary we deduce the boundary layer corrector from $\left(\mathbf{u}^{\varepsilon}, p^{\varepsilon}\right)$ and define

$\mathbf{U}^{\varepsilon}(x)=\mathbf{u}^{\varepsilon}(x)-\varepsilon P\left(x_{1}\right) \mathbf{w}(x / \varepsilon), P^{\varepsilon}(x)=p^{\varepsilon}(x)-\left[P\left(x_{1}\right) \pi(x / \varepsilon)+Q\left(x_{1}\right)\right]$.

The stress tensor $\mathbf{T}(\mathbf{v}, q)=2 \mu \mathbf{D v}-q \mathbf{I}$ for such approximation satisfies

$$
\begin{gathered}
\mathbf{T}\left(\mathbf{U}^{\varepsilon}, P^{\varepsilon}\right)=\mathbf{T}\left(\mathbf{u}^{\varepsilon}, p^{\varepsilon}\right)-P\left(x_{1}\right) \\
-2 \mu \varepsilon \frac{d P}{d x_{1}}\left[\begin{array}{cc}
w_{1} & w_{2} / 2 \\
w_{2} / 2 & 0
\end{array}\right]=\mathbf{T}\left(\mathbf{u}_{y} \mathbf{w}, p^{\varepsilon}\right)- \\
-\left[\begin{array}{cc}
P\left(x_{1}\right)\left(2 \mu \frac{\partial w_{1}}{\partial y_{1}}-\pi\right)+2 \mu \varepsilon \frac{d P}{d x_{1}} w_{1}-Q & \mu\left(P\left(x_{1}\right)\left(\frac{\partial w_{1}}{\partial y_{2}}+\frac{\partial w_{2}}{\partial y_{1}}\right)+\varepsilon \frac{d P}{d x_{1}} w_{2}\right) \\
\mu\left(P\left(x_{1}\right)\left(\frac{\partial w_{1}}{\partial y_{2}}+\frac{\partial w_{2}}{\partial y_{1}}\right)+\varepsilon \frac{d P}{d x_{1}} w_{2}\right) & P\left(x_{1}\right)\left(2 \mu \frac{\partial w_{2}}{\partial y_{2}}-\pi\right)-Q\left(x_{1}\right)
\end{array}\right]
\end{gathered}
$$


By direct computation we get

$$
\begin{gathered}
-\operatorname{div} \mathbf{T}\left(\mathbf{U}^{\varepsilon}, P^{\varepsilon}\right)=\mathbf{f}^{\varepsilon}, \\
\mathbf{f}^{\varepsilon} \equiv \mathbf{F}+\mu \varepsilon \frac{d^{2} P}{d x_{1}^{2}}\left(\mathbf{w}+w_{1} \mathbf{e}_{1}\right)+\frac{d P}{d x_{1}}\left(2 \mu \frac{\partial \mathbf{w}}{\partial y_{1}}-\pi \mathbf{e}_{1}+\mu \nabla_{y} w_{1}\right)-\frac{d Q}{d x_{1}} \mathbf{e}_{1}, \\
\operatorname{div} \mathbf{U}^{\varepsilon}=-\varepsilon \frac{d P}{d x_{1}} w_{1} \text { in } \Omega_{\varepsilon}, \\
\mathbf{U}^{\varepsilon}=0 \text { on } S_{\varepsilon}, \mathbf{U}^{\varepsilon}=-\varepsilon P\left(x_{1}\right) \mathbf{w}(x / \varepsilon) \text { on } \Gamma, \\
\left(-2 \mu \mathbf{D} \mathbf{U}^{\varepsilon}+P^{\varepsilon} \mathbf{I}\right) \mathbf{e}_{2}=0 \text { on } \Sigma .
\end{gathered}
$$

We want to derive appropriate a priori estimates for $\left(\mathbf{U}^{\varepsilon}, P^{\varepsilon}\right)$. However, according to (46), the divergence of $\mathbf{U}^{\varepsilon}$ is still too large for our purpose. Thus we need to compute the additional divergence corrector.

Lemma 3. There exists $\boldsymbol{\Phi} \in H^{2}\left(G^{*}\right)^{2}$ such that

$$
\begin{aligned}
& \operatorname{div}_{y} \mathbf{\Phi}=w_{1} \text { in } G^{*}, \\
& \mathbf{\Phi} \text { is 1-periodic in } y_{1}, \boldsymbol{\Phi}=0 \text { on } \mathcal{C}, \boldsymbol{\Phi}\left(y_{1}, 0\right)=C \mathbf{e}_{2}, \\
& e^{\gamma\left|y_{2}\right|} \mathbf{\Phi} \in L^{2}\left(G^{*}\right)^{4} \text { and }\left|\mathbf{\Phi}\left(y_{1}, y_{2}\right)\right| \leq C e^{-\gamma\left|y_{2}\right|} \text {, for some } \gamma>0 .
\end{aligned}
$$

Proof. We follow [18] and search for $\boldsymbol{\Phi}$ in the form

$$
\mathbf{\Phi}=\nabla_{y} \psi+\operatorname{curl}_{y} h=\left(\frac{\partial \psi}{\partial y_{1}}-\frac{\partial h}{\partial y_{2}}, \frac{\partial \psi}{\partial y_{2}}+\frac{\partial h}{\partial y_{1}}\right) .
$$

The function $\psi$ solves

$$
\begin{aligned}
& -\Delta_{y} \psi=w_{1}(y) \text { in } G^{*}, \frac{\partial \psi}{\partial \mathbf{n}}=0 \text { on } \mathcal{C} \\
& \frac{\partial \psi}{\partial y_{2}}=d_{0}=\text { const. for } y_{2}=0, \quad \psi \text { is } 1 \text {-periodic in } y_{1},
\end{aligned}
$$

with $\mathbf{n}=\left(n_{1}, n_{2}\right)$ being the exterior unit normal on $\mathcal{C}$ and $\mathbf{t}=\left(-n_{2}, n_{1}\right)$ the tangent. The constant $d_{0}$ is chosen in a way that problem (52)-(53) admits a solution. By simple integration it turns out that $d_{0}=-\int_{G^{*}} w_{1}(y) d y$. Since the right-hand side is in $H^{1}\left(G^{*}\right)$, the problem has a solution $\psi \in H^{3}\left(G^{*}\right)$ that can be chosen to have an exponential decay

$$
|\psi|_{H^{1}\left(G^{*} \cap\left\{\left|y_{2}\right|>s\right\}\right)} \leq C e^{-\gamma s} .
$$


Next we use the trace theorem and construct a $y_{1}$-periodic function $h \in H^{3}\left(G^{*}\right)$ such that

$$
\frac{\partial h}{\partial \mathbf{t}}=\operatorname{curl} h \cdot \mathbf{n}=0, \frac{\partial h}{\partial \mathbf{n}}=\operatorname{curl} h \cdot \mathbf{t}=-\frac{\partial \psi}{\partial \mathbf{t}} \quad \text { on } \mathcal{C}
$$

and

$\frac{\partial h}{\partial y_{2}}\left(y_{1}, 0\right)=\frac{\partial \psi}{\partial y_{1}}\left(y_{1}, 0\right), \frac{\partial h}{\partial y_{1}}\left(y_{1}, 0\right)=0$ (achieved if we take $h\left(y_{1}, 0\right)=$ const. $)$.

The function $\boldsymbol{\Phi}$, constructed above, satisfies (49) and (50). Exponential decay (54) of $\psi$ implies exponential decay of $h$ in the same sense and, finally, gives 51.

Again, assuming that $\mathbf{U}^{\varepsilon}$ is extended by zero to the pores $B_{\varepsilon}$ we extend $P^{\varepsilon}$ 175 using the formula 16 to prove:

\section{Lemma 4.}

$$
\begin{aligned}
& \left|\nabla \mathbf{U}^{\varepsilon}\right|_{L^{2}(\Omega)} \leq C \varepsilon \\
& \left|\mathbf{U}^{\varepsilon}\right|_{L^{2}(\Omega)} \leq C \varepsilon^{2} \\
& \left|P^{\varepsilon}\right|_{L^{2}(\Omega)} \leq C .
\end{aligned}
$$

Proof. It is straightforward to see that for the right-hand side, we have

$$
\left|\mathbf{f}^{\varepsilon}\right|_{L^{2}(\Omega)} \leq C
$$

Furthermore

$$
\mathbf{f}^{\varepsilon}=\mathbf{F}-\left(\frac{d P}{d x_{1}} C_{\pi}+\frac{d Q}{d x_{1}}\right) \mathbf{e}_{1}+\mathbf{g}^{\varepsilon},
$$

with $\left|\mathbf{g}^{\varepsilon}\right|_{L^{2}(\Omega)}=O(\sqrt{\varepsilon})$. The idea is to test the system 44 with

$$
\tilde{\mathbf{U}}^{\varepsilon}=\mathbf{U}^{\varepsilon}+\varepsilon^{2} \frac{d P}{d x_{1}}\left(x_{1}\right) \Phi\left(\frac{x}{\varepsilon}\right),
$$

where $\Phi$ is constructed in lemma 3 . By the construction

$$
\operatorname{div} \tilde{\mathbf{U}}^{\varepsilon}=\varepsilon^{2} \frac{d^{2} P}{d x_{1}^{2}} \Phi_{1}^{\varepsilon}
$$

with $\Phi^{\varepsilon}(x)=\Phi(x / \varepsilon)$. Thus

$$
\left|\operatorname{div} \tilde{\mathbf{U}}^{\varepsilon}\right|_{L^{2}(\Omega)} \leq C \varepsilon^{5 / 2}
$$


The weak form of 44 reads

$$
2 \mu \int_{\Omega_{\varepsilon}} \mathbf{D ~ U}^{\varepsilon}: \mathbf{D} \mathbf{z} d x-\int_{\Omega_{\varepsilon}} P^{\varepsilon} \operatorname{div} \mathbf{z} d x=\int_{\Omega_{\varepsilon}} \mathbf{f}^{\varepsilon} \mathbf{z} d x, \forall \mathbf{z} \in X_{2}^{\varepsilon}
$$

so that

$$
\left|\int_{\Omega_{\varepsilon}} P^{\varepsilon} \operatorname{div} \mathbf{z} d x\right| \leq C\left(\left|\mathbf{D ~ U}^{\varepsilon}\right|_{L^{2}\left(\Omega_{\varepsilon}\right)}+\varepsilon\right)|\mathbf{z}|_{H^{1}\left(\Omega_{\varepsilon}\right)}, \quad \forall \mathbf{z} \in X_{2}^{\varepsilon} .
$$

Next we use identity (17) to obtain the estimate

$$
\left|\int_{\Omega} \tilde{P}^{\varepsilon} \operatorname{div} \mathbf{z} d x\right|=\left|\int_{\Omega_{\varepsilon}} P^{\varepsilon} \operatorname{div}\left(R_{\varepsilon} \mathbf{z}\right) d x\right| \leq \frac{C}{\varepsilon}\left(\left|\mathbf{D} \mathbf{U}^{\varepsilon}\right|_{L^{2}\left(\Omega_{\varepsilon}\right)}+\varepsilon\right)|\mathbf{z}|_{H^{1}(\Omega)},
$$

$\forall \mathbf{z} \in X_{2}$. Since div : $X_{2} \rightarrow L^{2}(\Omega)$ is a surjective continuous operator, 61 yields

$$
\left|\tilde{P}^{\varepsilon}\right|_{L^{2}(\Omega)} \leq C\left(\varepsilon^{-1}\left|\mathbf{D} \mathbf{U}^{\varepsilon}\right|_{L^{2}\left(\Omega_{\varepsilon}\right)}+1\right) .
$$

Now we take $\mathbf{z}=\tilde{\mathbf{U}}^{\varepsilon}$ as a test function in 59. To be precise, we observe that $\tilde{\mathbf{U}}^{\varepsilon}$ is not exactly in $X_{2}^{\varepsilon}$ since it is not equal to zero for $x_{2}=-d$. But, that value is exponentially small, of order $e^{-\gamma / \varepsilon}$, so it can be easily corrected by lifting its boundary value by a negligibly small function. Thus, slightly abusing the notation, we consider it as an element of $X_{2}^{\varepsilon}$. Then, due to the 58

$$
\left|\int_{\Omega_{\varepsilon}} P^{\varepsilon} \operatorname{div} \tilde{\mathbf{U}}^{\varepsilon} d x\right|=\left|\varepsilon^{2} \int_{\Omega_{\varepsilon}} P^{\varepsilon} \frac{d^{2} P}{d x_{1}^{2}} \Phi_{1}^{\varepsilon} d x\right| \leq C \varepsilon\left|\mathbf{D} \mathbf{U}^{\varepsilon}\right|_{L^{2}\left(\Omega_{\varepsilon}\right)}+C \varepsilon^{2} .
$$

Consequently, we get (55)-(57) .

At this point we use the classical two-scale convergence (see e.g. [26, [1]). For readers' convenience we recall basic definitions and compactness results.

Let $Y=[0,1]^{2}$ and let $C_{\#}^{\infty}(Y)$ be the set of all $C^{\infty}$ functions defined on $Y$ and periodic with period 1 . We say that a sequence $\left(v^{\varepsilon}\right)_{\varepsilon>0}$, from $L^{2}(\Omega)$, twoscale converges to a function $v^{0} \in L^{2}(\Omega)$ if

$$
\lim _{\varepsilon \rightarrow 0} \int_{\Omega} v^{\varepsilon}(x) \psi\left(x, \frac{x}{\varepsilon}\right) d x \rightarrow \int_{\Omega} \int_{Y} v^{0}(x, y) \psi(x, y) d x d y,
$$

for any $\psi \in C_{0}^{\infty}\left(\Omega ; C_{\#}^{\infty}(Y)\right)$.

For such convergence we have the following compactness result from [1] and 26] that we shall need in the sequel 
Theorem 5. - Let $\left(v^{\varepsilon}\right)_{\varepsilon>0}$ be a bounded sequence in $L^{2}(\Omega)$. Then we can extract a subsequence that two-scale converges to some $v^{0} \in L^{2}(\Omega \times Y)$.

- Let $\left(v^{\varepsilon}\right)_{\varepsilon>0}$ be a sequence in $H^{1}(\Omega)$ such that $v^{\varepsilon}$ and $\varepsilon \nabla v^{\varepsilon}$ are bounded in $L^{2}(\Omega)$. Then, there exists a function $v^{0} \in L^{2}\left(\Omega ; H_{\#}^{1}(Y)\right)$ and a subsequence for which

$$
\begin{aligned}
& v^{\varepsilon} \rightarrow v^{0} \text { in two-scales, } \\
& \varepsilon \nabla v^{\varepsilon} \rightarrow \nabla_{y} v^{0} \text { in two-scales. }
\end{aligned}
$$

Lemma 5. Let $\left(\mathbf{U}^{\varepsilon}, P^{\varepsilon}\right)$ be the solution of the residual problem $(46)-(48)$. Then

$$
\begin{aligned}
& \varepsilon^{-2} \mathbf{U}^{\varepsilon} \rightarrow \mathbf{U}^{0} \text { in two-scales, } \\
& \varepsilon^{-1} \nabla \mathbf{U}^{\varepsilon} \rightarrow \nabla_{y} \mathbf{U}^{0} \text { in two-scales, } \\
& P^{\varepsilon} \rightarrow P^{0} \text { in two-scales, }
\end{aligned}
$$

where $\left(\mathbf{U}^{0}, P^{0}, Q^{0}\right) \in L^{2}\left(\Omega ; H_{\#}^{1}\left(Y^{*}\right)\right) \times H^{1}(\Omega) \times L^{2}\left(\Omega ; L^{2}\left(Y^{*}\right) / \mathbb{R}\right)$ is the solution of the two-scale problem

$$
\begin{aligned}
& -\mu \Delta_{y} \mathbf{U}^{0}+\nabla_{y} Q^{0}+\nabla_{x} P^{0}=\mathbf{F}-\left(\frac{d Q}{d x_{1}}+C_{\pi} \frac{d P}{d x_{1}}\right) \mathbf{e}_{1} \text { in } Y^{*} \times \Omega, \\
& d i v_{y} \mathbf{U}^{0}=0 \text { in } Y^{*} \times \Omega, \\
& \mathbf{U}^{0}=0 \text { on } S \times \Omega,(70) \\
& \operatorname{div}_{x}\left(\int_{Y} \mathbf{U}^{0} d y\right)=0 \text { in } \Omega,\left(Q_{Y} \mathbf{U}^{0} d y\right) \cdot \mathbf{n}=0 \text { on } \Gamma, P^{0}=0 \text { on } 1-\text { periodic in } y,
\end{aligned}
$$

Proof. Using the estimates 55 - $(57)$ we get that there exist $\mathbf{U}^{0} \in L^{2}\left(\Omega ; H_{\#}^{1}(Y)\right)$ and $P^{0} \in L^{2}(\Omega \times Y)$ such that

$$
\begin{aligned}
& \varepsilon^{-2} \mathbf{U}^{\varepsilon} \rightarrow \mathbf{U}^{0} \text { in two-scales, } \\
& \varepsilon^{-1} \nabla \mathbf{U}^{\varepsilon} \rightarrow \nabla_{y} \mathbf{U}^{0} \text { in two-scales, } \\
& P^{\varepsilon} \rightarrow P^{0} \text { two-scale. }
\end{aligned}
$$

It follows directly that $\mathbf{U}^{0}(x, y)=0$ for $y \in \bar{A}$. 
First, for $\psi(x, y) \in C^{\infty}(Y \times \Omega)$, periodic in $y$, such that $\psi=0$ for $y \in \bar{A}$

$$
\begin{aligned}
& 0 \leftarrow \int_{\Omega} \frac{d P}{d x_{1}}\left(x_{1}\right) w_{1}\left(x, \frac{x}{\varepsilon}\right) \psi\left(x, \frac{x}{\varepsilon}\right) d x=\varepsilon^{-1} \int_{\Omega} \operatorname{div} \mathbf{U}^{\varepsilon} \psi\left(x, \frac{x}{\varepsilon}\right) d x \\
& =-\int_{\Omega}\left(\varepsilon \nabla_{x} \psi\left(x, \frac{x}{\varepsilon}\right)+\nabla_{y} \psi\left(x, \frac{x}{\varepsilon}\right)\right) \cdot \frac{\mathbf{U}^{\varepsilon}(x)}{\varepsilon^{2}} d x \rightarrow \\
& \rightarrow \int_{\Omega} \int_{Y} \mathbf{U}^{0} \cdot \nabla_{y} \psi(x, y) d y d x \Rightarrow \operatorname{div}_{y} \mathbf{U}^{0}=0 .
\end{aligned}
$$

We then test equations $44-48$ with $\mathbf{m}^{\varepsilon}(x)=\mathbf{m}\left(x, \frac{x}{\varepsilon}\right)$, where $\mathbf{m} \in H^{1}\left(\Omega ; H_{\#}^{1}(Y)\right)$,

195 $\mathbf{m}=0$ for $y \in \bar{M}$.

$$
\begin{aligned}
& 0 \leftarrow \varepsilon \int_{\Omega} \mathbf{f}^{\varepsilon} \mathbf{m}^{\varepsilon} d x=2 \mu \int_{\Omega} \mathbf{D} \mathbf{U}^{\varepsilon}(x)\left[\mathbf{D}_{y} \mathbf{m}\left(x, \frac{x}{\varepsilon}\right)+\varepsilon \mathbf{D}_{x} \mathbf{m}\left(x, \frac{x}{\varepsilon}\right)\right] d x- \\
& \int_{\Omega} P^{\varepsilon}(x)\left(\varepsilon \operatorname{div}_{x} \mathbf{m}(x, x / \varepsilon)+\operatorname{div}_{y} \mathbf{m}(x, x / \varepsilon)\right) d x \rightarrow \\
& -\int_{\Omega} \int_{Y} P^{0}(x, y) \operatorname{div}_{y} \mathbf{m}(x, y) d y d x .
\end{aligned}
$$

Thus $\nabla_{y} P^{0}=0$ implying $P^{0}=P^{0}(x)$.

Next we test system 44 - 48 with $\mathbf{Z}^{\varepsilon}(x)=\mathbf{Z}\left(x, \frac{x}{\varepsilon}\right)$, where $\mathbf{Z} \in H^{1}\left(\Omega ; H_{\#}^{1}(Y)\right)$, such that $\operatorname{div}_{y} \mathbf{Z}=0$ and $\mathbf{Z}=0$ for $y \in \bar{A}$. It yields

$$
\begin{aligned}
& \int_{\Omega}\left[\mathbf{F}-\frac{d P\left(x_{1}\right) C_{\pi}+Q\left(x_{1}\right)}{d x_{1}} \mathbf{e}_{1}\right] \int_{Y} \mathbf{Z} d y \leftarrow \int_{\Omega} \mathbf{f}^{\varepsilon} \mathbf{Z}^{\varepsilon}=-\int_{\Omega} P^{\varepsilon}(x) \operatorname{div}_{x} \mathbf{Z}(x, x / \varepsilon) d x \\
& +\frac{2 \mu}{\varepsilon} \int_{\Omega} \mathbf{D} \mathbf{U}^{\varepsilon}(x)\left(\mathbf{D}_{y} \mathbf{Z}\left(x, \frac{x}{\varepsilon}\right)+\varepsilon \mathbf{D}_{x} \mathbf{Z}\left(x, \frac{x}{\varepsilon}\right)\right) d x \rightarrow \\
& \quad \rightarrow 2 \mu \int_{\Omega} \int_{Y} \mathbf{D}_{y} \mathbf{U}^{0}(x, y) \mathbf{D}_{y} \mathbf{Z}(x, y) d y d x-\int_{\Omega} \int_{Y} P^{0}(x) \operatorname{div}_{x} \mathbf{Z}(x, y) d y d x .
\end{aligned}
$$

200 We conclude that $\nabla_{x} P^{0} \in L^{2}(\Omega)$ and $\left(\mathbf{U}^{0}, P^{0}\right)$ satisfies equations 69- 71 .

The effective filtration velocity boundary conditions are determined by picking a smooth test-function $\psi \in C^{\infty}(\Omega)$, periodic in $x_{1}, \psi=0$ on $\Sigma$, and testing

$$
\operatorname{div} \tilde{\mathbf{U}}^{\varepsilon}=\varepsilon^{2} \frac{d P^{\prime}}{d x_{1}} \Phi_{1}^{\varepsilon}
$$


with it. It gives

$$
\begin{aligned}
& -\int_{\Omega_{\varepsilon}} \frac{d P^{\prime}}{d x_{1}}\left(x_{1}\right) \Phi_{1}\left(\frac{x}{\varepsilon}\right) \psi(x) d x=\varepsilon^{-2} \int_{\Omega_{\varepsilon}} \operatorname{div} \tilde{\mathbf{U}}^{\varepsilon}(x) \psi(x) d x= \\
& =-\int_{\Omega_{\varepsilon}} \varepsilon^{-2} \tilde{\mathbf{U}}^{\varepsilon}(x) \cdot \nabla \psi(x) d x-\int_{0}^{\ell} \tilde{U}_{2}^{\varepsilon}\left(x_{1},-d\right) \psi\left(x_{1},-d\right) d x_{1} .
\end{aligned}
$$

The last integral on the right hand side is negligible due to the exponential decay of $\mathbf{w}$ and $\boldsymbol{\Phi}$. The first integral on the right hand side, due to (66), converges and, due to the construction of $\tilde{\mathbf{U}}^{\varepsilon}$,

$$
\begin{aligned}
\lim _{\varepsilon \rightarrow 0} \int_{\Omega} \varepsilon^{-2} \tilde{\mathbf{U}}^{\varepsilon}(x) \cdot \nabla \psi(x) d x & =\lim _{\varepsilon \rightarrow 0} \int_{\Omega} \varepsilon^{-2} \mathbf{U}^{\varepsilon}(x) \cdot \nabla \psi(x) d x= \\
& =\int_{\Omega} \int_{Y} \mathbf{U}^{0}(x, y) d y \cdot \nabla \psi(x) d x .
\end{aligned}
$$

For the left-hand side in $(76)$ we get

$$
\left|\int_{\Omega} \frac{d^{2} P}{d x_{1}^{2}}\left(x_{1}\right) \Phi_{1}\left(\frac{x}{\varepsilon}\right) \psi(x) d x\right| \leq C \sqrt{\varepsilon} .
$$

Thus

$$
\int_{\Omega}\left(\int_{Y} \mathbf{U}^{0} d y\right) \cdot \nabla \psi d x=0
$$

meaning that

$$
\operatorname{div}_{x}\left(\int_{Y} \mathbf{U}^{0} d y\right)=0 \text { in } \Omega,\left(\int_{Y} \mathbf{U}^{0} d y\right) \cdot \mathbf{n}=0 \text { on } \Gamma .
$$

We still need to determine the boundary condition for $P^{0}$ on $\Sigma$.

Let $\mathbf{b}$ be a smooth function defined on $\Omega \times Y$, such that $\operatorname{div}_{y} \mathbf{b}=0$ and $\mathbf{b}=0$ on $\Gamma$ and $\mathbf{b}=0$ for $y \in \bar{A}$. We now use $\mathbf{b}^{\varepsilon}(x)=\mathbf{b}(x, x / \varepsilon)$ as a test function in (44)- (48). We obtain

$$
\begin{gathered}
\int_{\Omega} \mathbf{f}^{\varepsilon} \cdot \mathbf{b}^{\varepsilon} d x=2 \mu \int_{\Omega} \mathbf{D} \mathbf{U}^{\varepsilon}\left[\mathbf{D}_{x} \mathbf{b}\left(\cdot, \frac{\cdot}{\varepsilon}\right)+\varepsilon^{-1} \mathbf{D}_{y} \mathbf{b}\left(\cdot, \frac{\cdot}{\varepsilon}\right)\right] d x- \\
\int_{\Omega} P^{\varepsilon} \operatorname{div}_{x} \mathbf{b}\left(\cdot, \frac{\dot{\varepsilon}}{\varepsilon}\right) d x \rightarrow 2 \mu \int_{\Omega} \int_{Y} \mathbf{D}_{y} \mathbf{U}^{0} \mathbf{D}_{y} \mathbf{b} d y d x-\int_{\Omega} P^{0} \operatorname{div}_{x}\left(\int_{Y} \mathbf{b} d y\right) d x .
\end{gathered}
$$

As for the left-hand side, we have

$$
\int_{\Omega} \mathbf{f}^{\varepsilon} \cdot \mathbf{b}^{\varepsilon} d x \rightarrow \int_{\Omega}\left[\mathbf{F}-\frac{d\left(P\left(x_{1}\right) C_{\pi}+Q\left(x_{1}\right)\right)}{d x_{1}} \mathbf{e}_{1}\right]\left(\int_{Y} \mathbf{b} d y\right) d x
$$


so that

$$
\begin{gathered}
2 \mu \int_{\Omega} \int_{Y} \mathbf{D}_{y} \mathbf{U}^{0} \mathbf{D}_{y} \mathbf{b} d y d x-\int_{\Omega} P^{0} \operatorname{div}_{x}\left(\int_{Y} \mathbf{b} d y\right) d x= \\
\int_{\Omega} \int_{Y} \mathbf{b}\left[\mathbf{F}-\frac{d\left(P\left(x_{1}\right) C_{\pi}+Q\left(x_{1}\right)\right)}{d x_{1}} \mathbf{e}_{1}\right] d y d x .
\end{gathered}
$$

Using 69)- 72 yields

$$
\int_{\Omega} P^{0} \operatorname{div}\left(\int_{Y} \mathbf{b} d y\right) d x=-\int_{\Omega} \nabla P^{0} \cdot\left(\int_{Y} \mathbf{b} d y\right) d x
$$

It implies

$$
2 \mu \int_{\Sigma}\left(\int_{Y} \mathbf{b} \cdot \mathbf{e}_{2} d y\right) P^{0} d x=0
$$

and, finally, $P^{0}=0$ on $\Sigma$.

Proving uniqueness of a weak solution for problem 69 - -72 is straightforward.

\subsection{Step four: Strong convergence}

We start by proving the strong convergence for the pressure. We follow the approach from [27]. Let $\left\{\mathbf{z}^{\varepsilon}\right\}_{\varepsilon>0}$ be a sequence in $X_{2}$ such that

$$
\mathbf{z}^{\varepsilon} \rightarrow \mathbf{z}^{0} \quad \text { weakly in } H^{1}(\Omega)
$$

Then we have

$$
\int_{\Omega} \tilde{P}^{\varepsilon} \operatorname{div} \mathbf{z}^{\varepsilon} d x-\int_{\Omega} P^{0} \operatorname{div} \mathbf{z} d x=\int_{\Omega} \tilde{P}^{\varepsilon} \operatorname{div}\left(\mathbf{z}^{\varepsilon}-\mathbf{z}\right) d x+\int_{\Omega}\left(\tilde{P}^{\varepsilon}-P^{0}\right) \operatorname{div} \mathbf{z} d x .
$$

For two integrals on the right-hand side we have

$$
\begin{aligned}
& \lim _{\varepsilon \rightarrow 0} \int_{\Omega}\left(\tilde{P}^{\varepsilon}-P^{0}\right) \operatorname{div} \mathbf{z} d x=0 \text { and } \\
& \int_{\Omega} \tilde{P}^{\varepsilon} \operatorname{div}\left(\mathbf{z}^{\varepsilon}-\mathbf{z}\right) d x=\int_{\Omega_{\varepsilon}} P^{\varepsilon} \operatorname{div} R_{\varepsilon}\left(\mathbf{z}^{\varepsilon}-\mathbf{z}\right) d x= \\
& 2 \mu \int_{\Omega_{\varepsilon}} \mathbf{D}\left(\frac{\mathbf{U}^{\varepsilon}}{\varepsilon}\right) \varepsilon \mathbf{D}\left(R_{\varepsilon}\left(\mathbf{z}^{\varepsilon}-\mathbf{z}\right)\right) d x \rightarrow 0 \text { as } \varepsilon \rightarrow 0 .
\end{aligned}
$$

Using surjectivity of the operator div $: X_{2} \rightarrow L^{2}(\Omega)$ we conclude that $\tilde{P}^{\varepsilon} \rightarrow P^{0}$ strongly in $L^{2}(\Omega)$. 
Next we prove the strong convergence for the velocity. We define

$$
\mathbf{U}^{0, \varepsilon}(x)=\sum_{k=1}^{2} \mathbf{w}^{k}(x / \varepsilon)\left[F_{k}(x)-\frac{\partial}{\partial x_{k}}\left(P^{0}(x)+C_{\pi} P\left(x_{1}\right)+Q\left(x_{1}\right)\right)\right] .
$$

Then for the $L^{2}$-norms we have

$$
\begin{aligned}
& \int_{\Omega_{\varepsilon}}\left|\frac{\mathbf{U}^{\varepsilon}}{\varepsilon^{2}}-\mathbf{U}^{0, \varepsilon}\right|^{2} d x \leq C 2 \mu \varepsilon^{2} \int_{\Omega_{\varepsilon}}\left|\mathbf{D}\left(\frac{\mathbf{U}^{\varepsilon}}{\varepsilon^{2}}-\mathbf{U}^{0, \varepsilon}\right)\right|^{2} d x= \\
& =C\left\{2 \mu \varepsilon^{-2} \int_{\Omega_{\varepsilon}}\left|\mathbf{D} \mathbf{U}^{\varepsilon}\right|^{2} d x+2 \mu \varepsilon^{2} \int_{\Omega_{\varepsilon}}\left|\mathbf{D} \mathbf{U}^{0, \varepsilon}\right|^{2} d x-\right. \\
& \left.-4 \mu \int_{\Omega_{\varepsilon}} \mathbf{D}\left(\frac{\mathbf{U}^{\varepsilon}}{\varepsilon}\right) \varepsilon \mathbf{D} \mathbf{U}^{0, \varepsilon} d x\right\} .
\end{aligned}
$$

Using the smoothness of $\mathbf{U}^{0}$ we get, as $\varepsilon \rightarrow 0$

(i) $\varepsilon^{2} \int_{\Omega_{\varepsilon}}\left|\mathbf{D} \mathbf{U}^{0, \varepsilon}\right|^{2} d x=\int_{\Omega_{\varepsilon}}\left|\mathbf{D}_{y} \mathbf{U}^{0, \varepsilon}\right|^{2} d x+O(\varepsilon) \rightarrow \int_{\Omega \times Y^{*}}\left|\mathbf{D}_{y} \mathbf{U}^{0}\right|^{2} d x d y$.

(ii) $2 \mu \int_{\Omega \times Y^{*}}\left|\mathbf{D}_{y} \mathbf{U}^{0}\right|^{2} d x=\int_{\Omega}\left(\mathbf{F}-\frac{d\left(P\left(x_{1}\right) C_{\pi}+Q\left(x_{1}\right)\right)}{d x_{1}} \mathbf{e}_{1}\right) \int_{Y^{*}} \mathbf{U}^{0} d y d x$.

(iii) $2 \mu \varepsilon^{-2} \int_{\Omega_{\varepsilon}}\left|\mathbf{D} \mathbf{U}^{\varepsilon}\right|^{2} d x=2 \mu \varepsilon^{-2} \int_{\Omega_{\varepsilon}} \mathbf{D} \mathbf{U}^{\varepsilon} \mathbf{D} \tilde{\mathbf{U}}^{\varepsilon} d x+O(\sqrt{\varepsilon})$.

(iv)

$$
\begin{gathered}
2 \mu \varepsilon^{-2} \int_{\Omega_{\varepsilon}} \mathbf{D} \mathbf{U}^{\varepsilon} \mathbf{D} \tilde{\mathbf{U}}^{\varepsilon} d x-\varepsilon^{-2} \int_{\Omega_{\varepsilon}} P^{\varepsilon} \operatorname{div} \tilde{\mathbf{U}}^{\varepsilon} d x= \\
\int_{\Omega_{\varepsilon}}\left(\mathbf{F}-\frac{d\left(P\left(x_{1}\right) C_{\pi}+Q\left(x_{1}\right)\right)}{d x_{1}} \mathbf{e}_{1}\right) \frac{\mathbf{U}^{\varepsilon}}{\varepsilon^{2}} d x+O(\sqrt{\varepsilon}) .
\end{gathered}
$$

(v) $\varepsilon^{-2} \int_{\Omega_{\varepsilon}} P^{\varepsilon} \operatorname{div} \tilde{\mathbf{U}}^{\varepsilon} d x=\int_{\Omega_{\varepsilon}} P^{\varepsilon} \frac{d^{2} P}{d x_{1}^{2}} \boldsymbol{\Phi}^{\varepsilon} d x \rightarrow 0$.

(vi)

$$
\begin{aligned}
& (i i i),(i v) \text { and }(v) \Rightarrow \\
& 2 \mu \varepsilon^{-2} \int_{\Omega_{\varepsilon}}\left|\mathbf{D} \mathbf{U}^{\varepsilon}\right|^{2} d x \rightarrow \int_{\Omega}\left[\mathbf{F}-\frac{d\left(P\left(x_{1}\right) C_{\pi}+Q\left(x_{1}\right)\right)}{d x_{1}} \mathbf{e}_{1}\right] \int_{Y^{*}} \mathbf{U}^{0} d y d x . \\
& { }_{225}^{22} \quad \text { (vii) } \int_{\Omega_{\varepsilon}} \mathbf{D}\left(\frac{\mathbf{U}^{\varepsilon}}{\varepsilon}\right) \varepsilon \mathbf{D} \mathbf{U}^{0, \varepsilon} d x \rightarrow \int_{\Omega \times Y^{*}}\left|\mathbf{D}_{y} \mathbf{U}^{0}\right|^{2} d x d y \text {. }
\end{aligned}
$$


Thus

$$
\lim _{\varepsilon \rightarrow 0} \int_{\Omega_{\varepsilon}}\left|\frac{\mathbf{U}^{\varepsilon}}{\varepsilon^{2}}-\mathbf{U}^{0, \varepsilon}\right|^{2} d x=0
$$

\subsection{Step five: Weak* convergence of the boundary layer corrector}

To prove convergence 12 we need to show that

$$
\varepsilon^{-1} P\left(x_{1}\right) \mathbf{w}(x / \varepsilon) \rightarrow P\left(x_{1}\right)\left(\int_{G^{*}} \mathbf{w}(y) d y\right) \delta_{\Sigma} \text { weak* in } \mathcal{M}(\Omega) .
$$

Thus we take the test function $\mathbf{z} \in C(\bar{\Omega})^{2}$ and, using the exponential decay of ${ }_{230} \mathbf{w}$, we get

$$
\begin{gathered}
\frac{1}{\varepsilon} \int_{\Omega} P\left(x_{1}\right) \mathbf{w}\left(\frac{x}{\varepsilon}\right) \mathbf{z}(x) d x=\frac{1}{\varepsilon} \int_{0}^{\ell} P\left(x_{1}\right) \int_{\varepsilon \log \varepsilon}^{0} \mathbf{w}\left(\frac{x}{\varepsilon}\right) \mathbf{z}(x) d x_{2} d x_{1}+O(\varepsilon)= \\
=\int_{0}^{\ell} P\left(x_{1}\right) \mathbf{z}\left(x_{1}, 0\right) \int_{-\infty}^{0} \mathbf{w}\left(\frac{x_{1}}{\varepsilon}, y_{2}\right) d y_{2} d x_{1}+O(\varepsilon|\log \varepsilon|) .
\end{gathered}
$$

Using the well known property of the mean of a periodic function (see e.g. [27]) yields

$$
\begin{aligned}
& \lim _{\varepsilon \rightarrow 0} \int_{0}^{\ell} P\left(x_{1}\right) \mathbf{z}\left(x_{1}, 0\right) \int_{-\infty}^{0} \mathbf{w}\left(\frac{x_{1}}{\varepsilon}, y_{2}\right) d y_{2} d x_{1}= \\
& =\int_{0}^{\ell} P\left(x_{1}\right) \mathbf{z}\left(x_{1}, 0\right)\left(\int_{-\infty}^{0} \int_{0}^{1} \mathbf{w}(y) d y_{1} d y_{2}\right) d x_{1}= \\
& =\int_{0}^{\ell} P\left(x_{1}\right) \mathbf{z}\left(x_{1}, 0\right) \int_{G^{*}} \mathbf{w}(y) d y d x_{1}=\left(\int_{G^{*}} \mathbf{w}(y) d y\right) P\left(x_{1}\right)\left\langle\delta_{\Sigma} \mid \mathbf{z}\right\rangle .
\end{aligned}
$$

4.6. Step six: Separation of scales and the end of the proof of Theorem 1

We can separate the variables in $69-72$ by setting

$$
\begin{aligned}
& \mathbf{U}^{0}(x, y)=\sum_{k=1}^{2} \mathbf{w}^{k}(y)\left[F_{k}(x)-\frac{\partial}{\partial x_{k}}\left(Q\left(x_{1}\right)+C_{\pi} P\left(x_{1}\right)+P^{0}(x)\right)\right], \\
& Q^{0}(x, y)=\sum_{k=1}^{2} \pi^{k}(y)\left[F_{k}(x)-\frac{\partial}{\partial x_{k}}\left(Q\left(x_{1}\right)+C_{\pi} P\left(x_{1}\right)+P^{0}(x)\right)\right],
\end{aligned}
$$

235 with

$$
\begin{aligned}
& -\mu \Delta \mathbf{w}^{k}+\nabla \pi^{k}=\mathbf{e}_{k}, \operatorname{div} \mathbf{w}^{k}=0 \text { in } Y^{*}, \\
& \mathbf{w}^{k}=0 \text { on } S,\left(\mathbf{w}^{k}, \pi^{k}\right) \text { is } 1-\text { periodic. }
\end{aligned}
$$


Inserting the separation of scales formulas 78$)-(79)$ into 69$)-(72)$ yields

$$
\left\{\begin{array}{l}
\operatorname{div} \mathbf{K}\left[\mathbf{F}-\nabla\left(P^{0}+C_{\pi} P+Q\right)\right]=0 \text { in } \Omega \\
P^{0}=0 \quad \text { on } \quad \Sigma, \quad P^{0} \quad \text { is } \ell-\text { periodic in } x_{1}, \\
\mathbf{n} \cdot \mathbf{K}\left[\mathbf{F}-\nabla\left(P^{0}+C_{\pi} P+Q\right)\right]=0 \text { on } \Gamma .
\end{array} .\right.
$$

Here

$$
\mathbf{K}=\left[K_{i j}\right]=\left[\int_{Y} w_{j}^{i} d y\right]
$$

stands for the positive definite and symmetric permeability tensor. System 82 a well-posed mixed boundary value problem for a linear elliptic equation for $P^{0}$. Nevertheless, it is important to note that $P^{0}$ is not the limit or homogenized pressure since

$$
p^{\varepsilon}(x)=P^{\varepsilon}(x)+\pi\left(\frac{x}{\varepsilon}\right) P\left(x_{1}\right)+Q\left(x_{1}\right) .
$$

Obviously

$$
p^{\varepsilon} \rightarrow p^{0} \equiv P^{0}+C_{\pi} P+Q .
$$

This ends the proof of theorem 1 since the limit pressure is $p^{0}$ and it satisfies the boundary value problem $(8)-10)$.

\section{Proof of Theorem 2}

We start by proving that problem (4)-(6) admits at least one solution satisfying estimates 18 - 20 .

It is well known that in the case of the stress boundary conditions, the inertia term poses difficulties and existence results for the stationary Navier-Stokes system can be obtained only under conditions on data and/or the Reynolds number (see e.g. [11]). Presence of many small solid obstacles in the porous media flows corresponds to a small Reynolds number, expressed through the presence of $\varepsilon$ in Poincaré's and trace estimates (14) and 15.

In order to estimate the inertia term we need fractional order Sobolev spaces. we recall that

$$
H^{1 / 2}(\Omega)^{2}=\left\{\mathbf{z} \in L^{2}(\Omega)^{2} \mid E \mathbf{z} \in H^{1 / 2}\left(\mathbb{R}^{2}\right)^{2}\right\},
$$


where $E: H^{1}(\Omega)^{2} \rightarrow H^{1}\left(\mathbb{R}^{2}\right)^{2}$ is the classical Sobolev extension map. It is defined on the spaces $H^{\alpha}(\Omega), \alpha \in(0,1)$ through interpolation (see [13], Chapter $6)$.

Next, after [13, Chapter 6, one has

$$
\left|\int_{\Omega_{\varepsilon}}\left(\mathbf{u}^{1, \varepsilon} \nabla\right) \mathbf{u}^{1, \varepsilon} \cdot \mathbf{v} d x\right| \leq C\left|\mathbf{u}^{1, \varepsilon}\right|_{H^{1 / 2}(\Omega)^{2}}\left|\nabla \mathbf{u}^{1, \varepsilon}\right|_{L^{2}(\Omega)^{2}}|\mathbf{v}|_{H^{1 / 2}(\Omega)^{2}}, \quad \forall \mathbf{v} \in V\left(\Omega_{\varepsilon}\right) .
$$

Using (14) in (84) yields

$$
\left|\int_{\Omega_{\varepsilon}}\left(\mathbf{u}^{1, \varepsilon} \nabla\right) \mathbf{u}^{1, \varepsilon} \cdot \mathbf{u}^{1, \varepsilon} d x\right| \leq C \varepsilon\left|\nabla \mathbf{u}^{1, \varepsilon}\right|_{L^{2}(\Omega)^{2}}^{3} .
$$

Now it is enough to have an a priori estimate for the $H^{1}$-norm. With such estimate the standard procedure would give existence of a solution. It consists of defining a finite dimensional Galerkin approximation and using the a priori estimate and Brouwer's theorem to show that it admits a solution satisfying a uniform $H^{1}$ - a priori estimate. Finally, we let the number of degrees of freedom in the Galerkin approximation tend to infinity and obtain a solution through the elementary compactness. For more details we refer to the textbook of Evans [14], subsection 9.1.

We recall that the variational form of (4)-(6) is

$$
\begin{gathered}
\left\langle\mathcal{L}^{\varepsilon} \mathbf{u}^{1, \varepsilon}, \mathbf{v}\right\rangle=2 \mu \int_{\Omega_{\varepsilon}} D \mathbf{u}^{1, \varepsilon}: D \mathbf{v} d x+\int_{\Omega_{\varepsilon}}\left(\mathbf{u}^{1, \varepsilon} \nabla\right) \mathbf{u}^{1, \varepsilon} \cdot \mathbf{v} d x- \\
-\int_{\Omega_{\varepsilon}} \mathbf{F} \cdot \mathbf{v} d x-\int_{\Sigma} \mathbf{H} \cdot \mathbf{v} d S=0, \quad \forall \mathbf{v} \in V\left(\Omega_{\varepsilon}\right) .
\end{gathered}
$$

Then, for $\varepsilon \leq \varepsilon_{0}$,

$$
\begin{gathered}
\left\langle\mathcal{L}^{\varepsilon} \mathbf{u}^{1, \varepsilon}, \mathbf{u}^{1, \varepsilon}\right\rangle \geq 2 \mu\left|D \mathbf{u}^{1, \varepsilon}\right|_{L^{2}\left(\Omega_{\varepsilon}\right)^{4}}^{2}-C \varepsilon\left|D \mathbf{u}^{1, \varepsilon}\right|_{L^{2}\left(\Omega_{\varepsilon}\right)^{4}}^{3}-C \sqrt{\varepsilon}\left|D \mathbf{u}^{1, \varepsilon}\right|_{L^{2}\left(\Omega_{\varepsilon}\right)^{4}} \geq \\
\geq \frac{C_{1}}{\varepsilon^{2}}>0, \quad \text { if }\left|D \mathbf{u}^{1, \varepsilon}\right|_{L^{2}\left(\Omega_{\varepsilon}\right)^{4}}=\frac{1}{\sqrt{\varepsilon}}
\end{gathered}
$$

As a direct consequence of (87), Brouwer's theorem implies existence of at least one solution for the $N$ dimensional Galerkin approximation corresponding to 86. (see [14, subsection 9.1). After passing to the limit $N \rightarrow+\infty$, we obtain existence of at least one solution $\mathbf{u}^{\varepsilon}$ for problem 86 , such that $\left|D \mathbf{u}^{1, \varepsilon}\right|_{L^{2}\left(\Omega_{\varepsilon}\right)^{4}} \leq$ 
$1 / \sqrt{\varepsilon}$. After plugging this information into estimate 85 , equation 86 yields the energy estimate

$$
2 \mu\left|D \mathbf{u}^{1, \varepsilon}\right|_{L^{2}\left(\Omega_{\varepsilon}\right)^{4}}^{2} \leq C \sqrt{\varepsilon}\left|D \mathbf{u}^{1, \varepsilon}\right|_{L^{2}\left(\Omega_{\varepsilon}\right)^{4}}^{2}+C \sqrt{\varepsilon}\left|D \mathbf{u}^{1, \varepsilon}\right|_{L^{2}\left(\Omega_{\varepsilon}\right)^{4}},
$$

implying estimates (18)-20).

Now we have

$$
\left|\int_{\Omega_{\varepsilon}}\left(\mathbf{u}^{1, \varepsilon} \nabla\right) \mathbf{u}^{1, \varepsilon} \cdot \mathbf{v}\right| \leq C \varepsilon\left|\nabla \mathbf{u}^{1, \varepsilon}\right|_{L^{2}(\Omega)^{2}}^{2}|\nabla \mathbf{v}|_{L^{2}(\Omega)^{2}} \leq C \varepsilon^{2}|\nabla \mathbf{v}|_{L^{2}(\Omega)^{2}}, \quad \forall \mathbf{v} \in V\left(\Omega_{\varepsilon}\right)
$$

and we conclude that in the calculations from subsections 4.24 .4 the inertia term does not play any role. Hence it does not contribute to the homogenized problem either. This observation concludes the proof of Theorem 2 .

\section{Numerical confirmation of the effective model}

In this section we use a direct computation of the boundary layer corrector (36-38) and the microscopic problem $(1-3)$ to numerically confirm the estimate 39

$$
\left|\pi-C_{\pi}\right|_{L^{2}\left(G^{*}\right)}=O(\sqrt{\epsilon})
$$

and the strong convergence of the effective pressure (7). For the pressure we find out

$$
\left|p^{\epsilon}-p^{0}\right|_{L^{2}(\Omega)}=O(\sqrt{\epsilon})
$$

which is consistent with the corrector type results from [18].

\subsection{Confirmation of boundary layer estimate}

We start with estimate (39). For this we need to compute the value $C_{\pi}$ which is the limit value of the boundary layer pressure $\pi$ for $y_{2} \rightarrow \infty$, see 41. Since the boundary layer problem is defined on an unbounded domain, we need to cut the domain and compute $C_{\pi, \mathrm{cut}}$, which is the approximation of $C_{\pi}$ on a cut-off domain with $\left|y_{2}\right|$ large enough so that the difference $\mid C_{\pi}-$ 
$C_{\pi, \mathrm{cut}} \mid$ is smaller than the machine precision. Since the value $\pi(\mathbf{y})$ stabilizes to $C_{\pi}$ exponentially fast, we expect that a boundary layer with a few unit cells gives an accurate approximation. Furthermore, the cut-off boundary layer is computed by the finite element method. Thus, we compute $C_{\pi, c u t}^{h}$, where the superscript $h$ indicates the Galerkin approximation, and we have to assure that the discretization error $\left|C_{\pi, \mathrm{cut}}-C_{\pi, \mathrm{cut}}^{h}\right|$ is small enough.

For the numerical approximation we first introduce the cut-off domain

$$
G_{l}^{*}:=G \backslash \bigcup_{j=0}^{-l}\left(j \mathbf{e}_{2}+A\right)
$$

and then consider the following cut-off boundary layer problem

Problem 1 (Cut-off boundary layer problem). Find $\mathbf{w}$ and $\pi$, both 1-periodic in $y_{1}$, such that it holds in the interior

$$
\begin{aligned}
-\mu \Delta_{y} \mathbf{w}_{l}+\nabla_{y} \pi_{l}=0 & \text { in } G_{l}^{*}, \\
\nabla \cdot \mathbf{w}_{l}=0 & \text { in } G_{l}^{*},
\end{aligned}
$$

and on the boundaries

$$
\begin{aligned}
\left(-2 \mu \mathbf{D}_{y} \mathbf{w}_{l}+\pi_{l} \mathbf{I}\right) & =\mathbf{e}_{1} & & \text { for } y_{2}=0, \\
\mathbf{w}_{l} & =0 & & \text { on } \mathcal{C} \\
w_{l, 2} & =\frac{\partial w_{l, 1}}{\partial y_{2}}=0 & & \text { on } \Gamma_{l},
\end{aligned}
$$

where $\Gamma_{l}=(0,1) \times l$ is the lower boundary of the cut-off domain.

The inclusions are defined as in Figure 1. The solid domain $A$ is

(a) circular in the isotropic case with radius $r=0.25$ and center $(0.5,0.5)$, see Figure 1a

(b) ellipsoidal in the anisotropic case with centre $(0.5,0.5)$ and semi-axes $a=$ 0.357142857 and $b=0.192307692$ rotated anti-clockwise by $45^{\circ}$, see Figure $1 \mathrm{~b}$. 


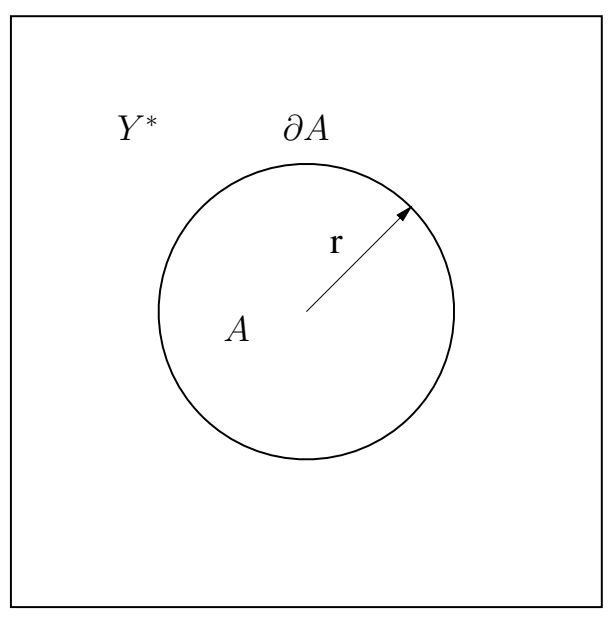

(a) Circles

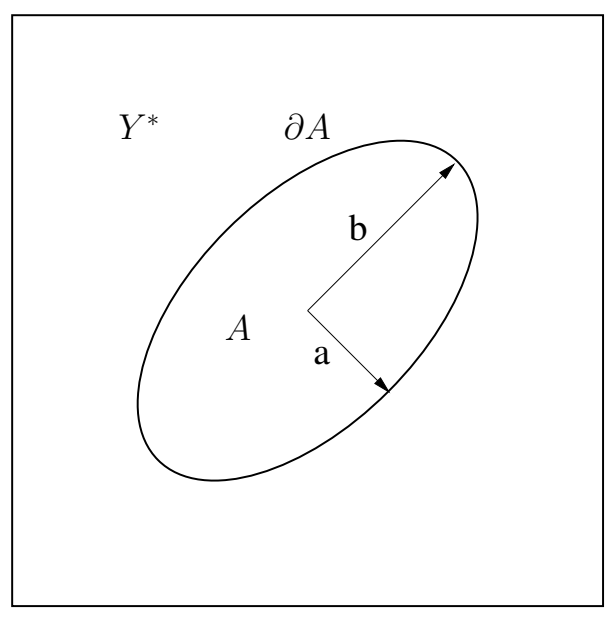

(b) Ellipses

Figure 1: Inclusion geometry.

Problem 89- 93 is approximated by the finite element method (FEM) reported. It can be observed that one inclusion is enough to get the exact value $C_{\pi}=0$ for the circular inclusions. In case of elliptical inclusions the pressure is stabilized for $l \geq 7$ and the effect of the cut-off domain can be seen only for smaller domains. Figure 2 shows a visualization of the boundary layer pressure 


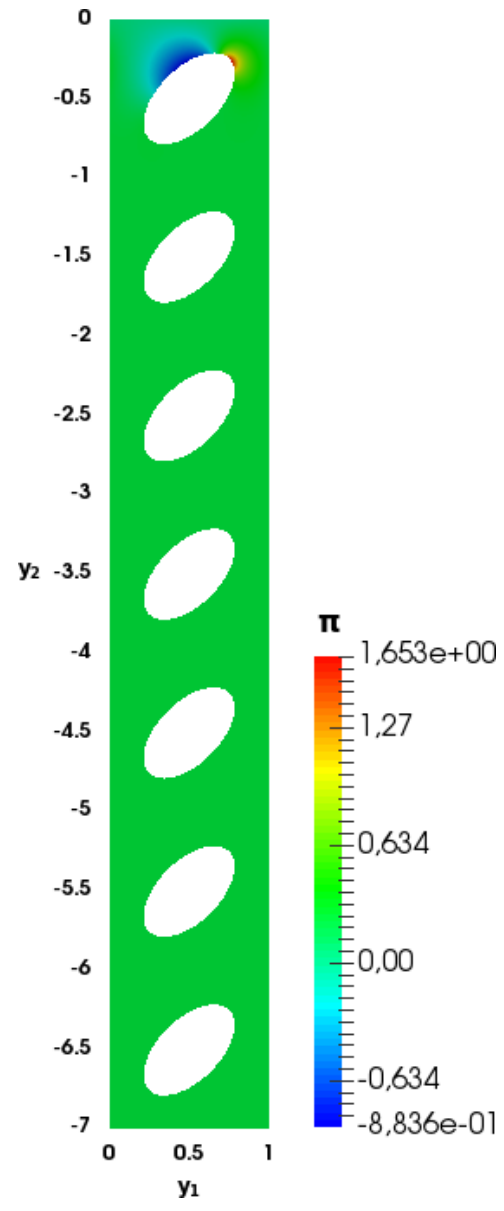

(a) Ellipses

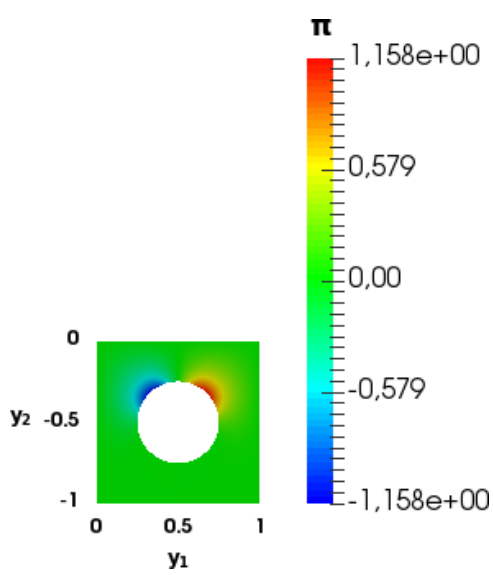

(b) Circles

Figure 2: Visualization of boundary layer pressure and cut-off domain. 


\begin{tabular}{ccc} 
\# inclusions & $C_{\pi}$ ellipses & $C_{\pi}$ circles \\
\hline 1 & 0.21632306467205 & 0 \\
2 & 0.21616431545737 & 0 \\
3 & 0.21616422652809 & 0 \\
4 & 0.21616422675581 & 0 \\
5 & 0.21616422675552 & 0 \\
6 & 0.21616422675555 & 0 \\
7 & 0.21616422675555 & 0
\end{tabular}

Table 1: Stabiliziation of $C_{\pi}$ in the cut-off domain with increasing number of inclusions. refined meshes have shown that the discretization error is of the order $O\left(10^{-8}\right)$. Therefore for the convergence study of the effective pressure, we consider as exact value for ellipses $C_{\pi}=0.2161642$.

After computing the constant $C_{\pi, \mathrm{cut}}^{h}$ we proceed with the confirmation of the estimate 39 and plot in Figure 3 the convergence curves. We confirm the expected convergence rate

$$
\left|\pi-C_{\pi}\right|_{L^{1}\left(G^{*}\right)}=O(\epsilon) \quad \text { and } \quad\left|\pi-C_{\pi}\right|_{L^{2}\left(G^{*}\right)}=O(\sqrt{\epsilon})
$$

\subsection{Confirmation of effective pressure values}

The next step is the confirmation of the estimate (7). For a stress tensor defined by the constant contact stress $(P, Q)$ and a right hand side which depends only on $x_{2}$ we have the analytical exact solution for the effective pressure

$$
p^{0}\left(x_{2}\right)=C_{\pi} P+Q-\int_{x_{2}}^{0} f_{2}(z) d z-\frac{K_{12}}{K_{22}} \int_{x_{2}}^{0} f_{1}(z) d z .
$$

To compute it we need the vales $K_{12}$ and $K_{22}$ of the permeability tensor. These are defined as follows

$$
K_{i j}:=\int_{Y^{*}} w_{c, j}^{i} d x
$$




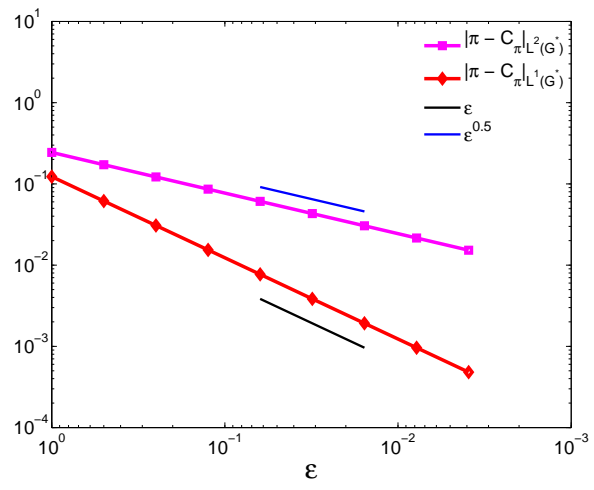

(a) Ellipses

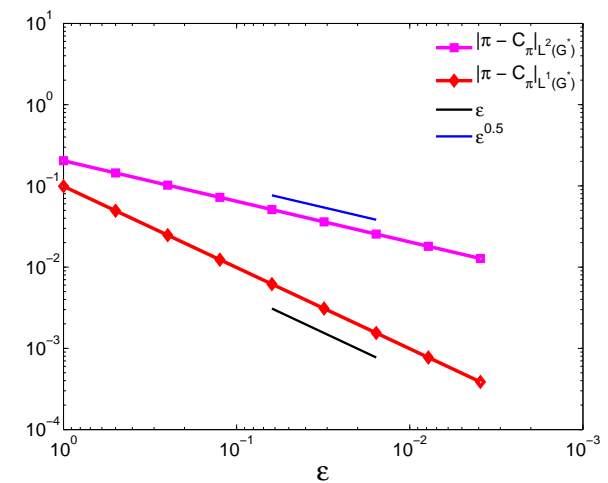

(b) Circles

Figure 3: Confirmation of convergence for the boundary layer problem.

\begin{tabular}{ccc} 
Inclusion type & $K_{12}$ & $K_{11}=K_{22}$ \\
\hline Ellipses & 0.00303449804138 & 0.0159787174788 \\
\hline Circles & 0.0 & 0.01990143534975
\end{tabular}

Table 2: Values of the permeability tensor components.

with the 1-periodic solution $\mathbf{w}_{\mathbf{c}}^{\mathbf{i}}(i=1,2)$ of the $i^{\text {th }}$ cell problem

$$
\begin{aligned}
-\Delta \mathbf{w}_{\mathbf{c}}^{\mathbf{i}}+\nabla \pi_{\mathbf{c}}^{\mathbf{i}}=\mathbf{e}_{i} & \text { in } Y^{*}, \\
\nabla \cdot \mathbf{w}_{c}^{i}=0 & \text { in } Y^{*}, \\
\mathbf{w}_{c}^{i}=0 & \text { on } \partial A
\end{aligned}
$$

where $Y^{*}$ is the unit pore domain of the cell problem with the corresponding inclusion $A$. The inclusions are defined as in our previous work 9]. They correspond to one cell of problem (89)- $(93)$ and they are shown on Figure 1 Therefore, we use the values of the permeability tensor computed therein and reported in Table 2. We use the extension $\tilde{p}_{h}^{\varepsilon}$ (16) for the microscopic pressure, where the subscript denotes the finite element approximation of the microscopic problem obtained with Taylor-Hood elements, as for the cut-off boundary layer. 


\begin{tabular}{ccccccc}
$\mu$ & $P\left(x_{1}\right)$ & $Q\left(x_{1}\right)$ & $f_{1}(\mathbf{x})$ & $f_{2}(\mathbf{x})$ & $C_{\pi}$ ellipses & $C_{\pi}$ circles \\
\hline 1 & 1 & 1 & 1 & 1 & 0.2161642 & 0
\end{tabular}

Table 3: Values used for the computations.

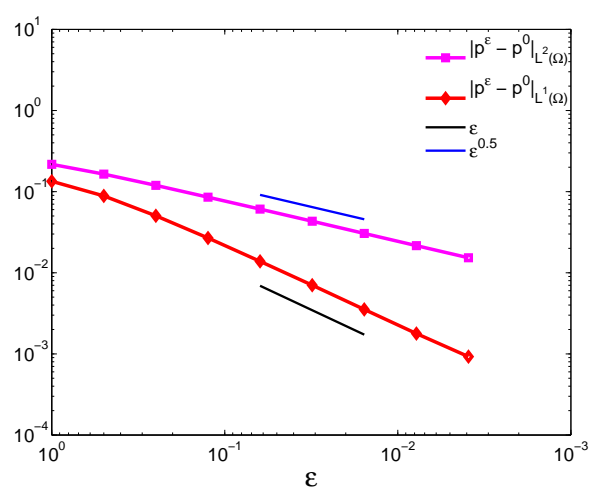

(a) Ellipses

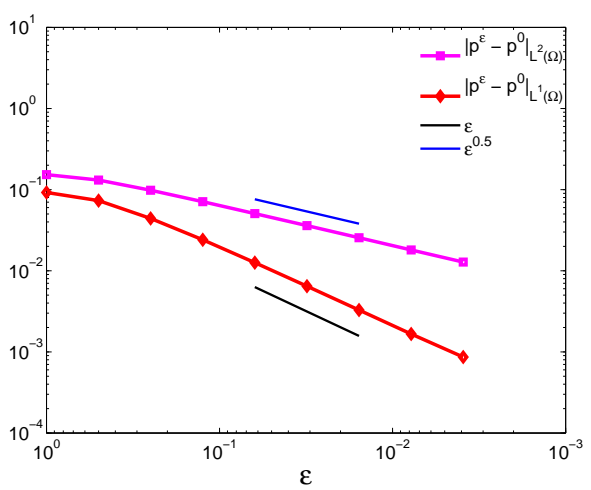

(b) Circles

Figure 4: Confirmation of convergence for the microscopic problem.

With the expression of the effective pressure and the extension pressure we compute the convergence estimates. For the test case we use the values $(P, Q)$ for the normal component of the stress tensor and $\mathbf{f}(\mathbf{x})$ for the right hand side, needed in formula (94), as reported in Table 3 . The results with the expected convergence rates are depicted in Figure 4. Finally, figures 5 and 6 show the velocity components, the velocity magnitude and the pressure in the microscopic problem for circles and ellipses. To simplify the visualization these figures show a microscopic problem with nine inclusions, so that the boundary layer is clearly visible.

\section{Conclusion}

The novelty of the result is in the boundary condition on $\Sigma$. The value of the Darcy pressure on the upper boundary $\Sigma$ is now prescribed and its value depends not only on the given applied pressure force $Q$ but also on the shear 


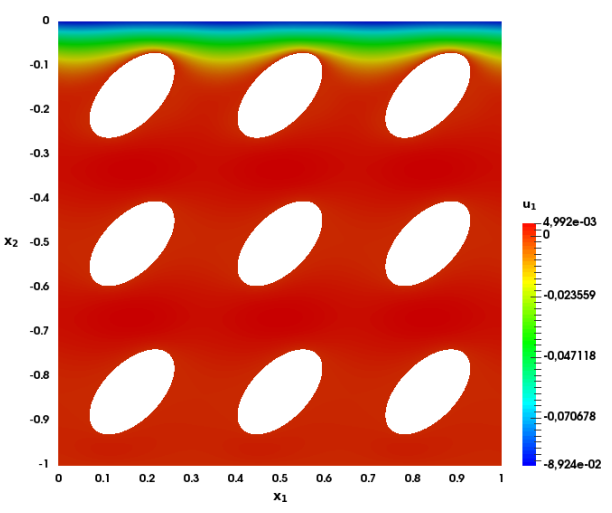

(a) $u_{1}$

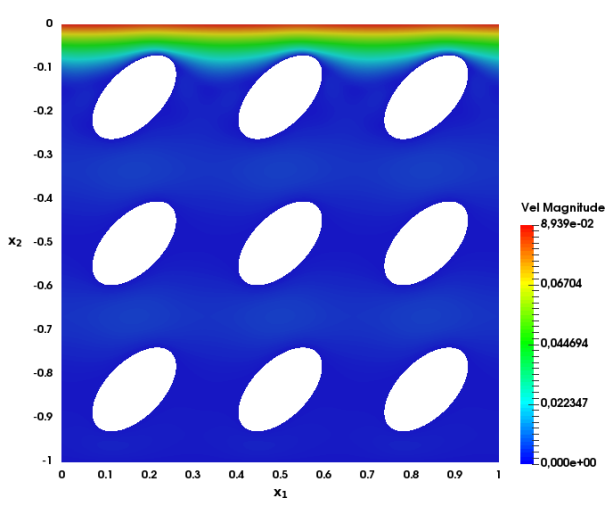

(c) $|\mathbf{u}|$

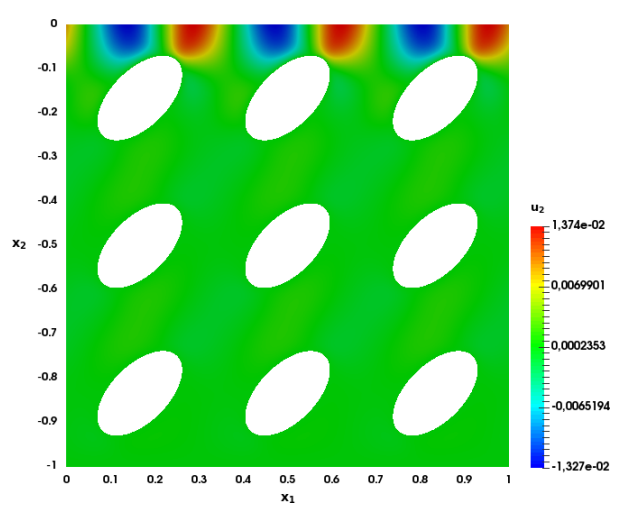

(b) $u_{2}$

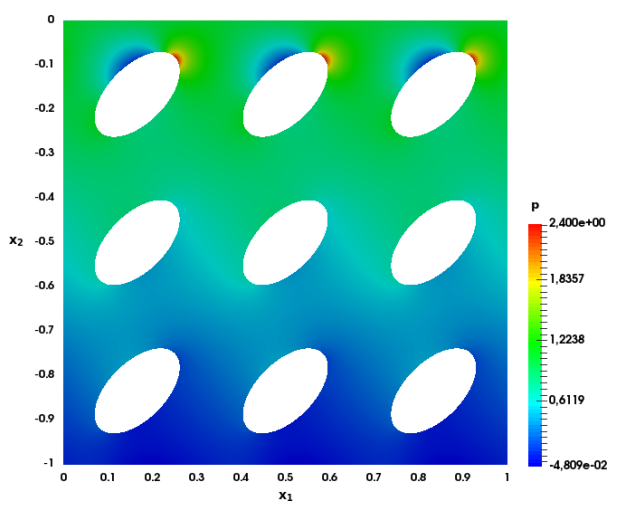

(d) $p$

Figure 5: Visualization of the microcopic velocity and pressure with elliptical inclusions. 


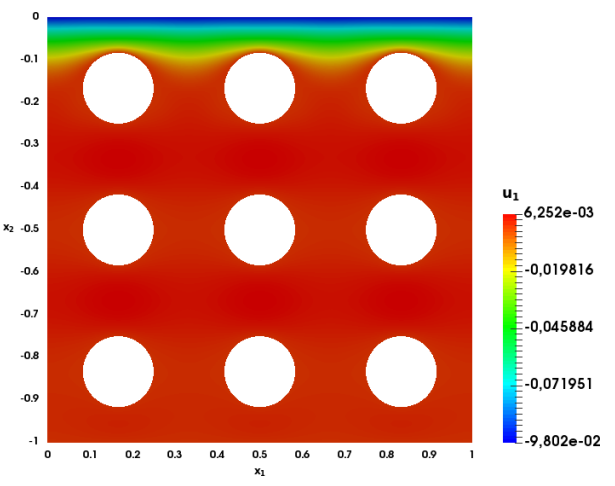

(a) $u_{1}$

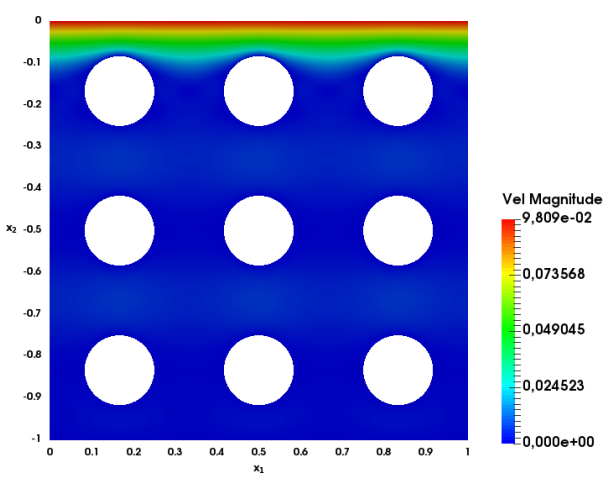

(c) $|\mathbf{u}|$

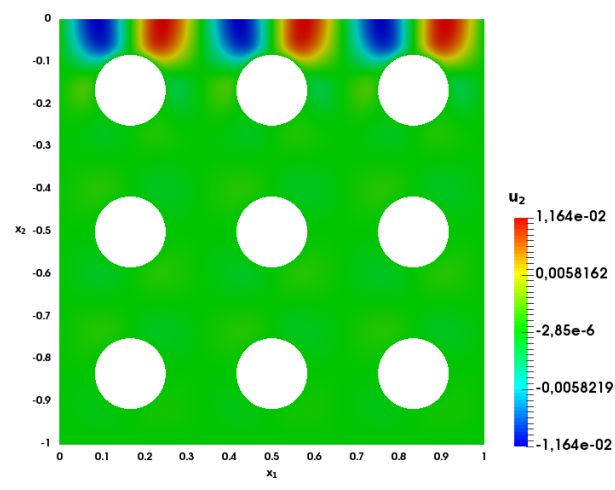

(b) $u_{2}$

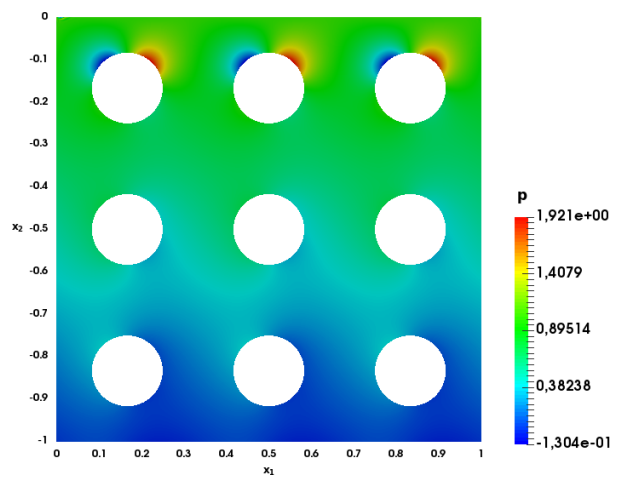

(d) $p$

Figure 6: Visualization of the microcopic velocity and pressure with circular inclusions. 
force $P$. Thus the macroscopic pressure is affected by the tangential component of the contact force and not only by the normal component. As for the velocity, it shows that

$$
\mathbf{u}^{\varepsilon}(x) \approx \varepsilon P\left(x_{1}\right) \mathbf{w}\left(\frac{x}{\varepsilon}\right)+\varepsilon^{2} \mathbf{V}(x) .
$$

Thus, in interior of the domain, the velocity is plain Darcean, while in vicinity of the upper boundary, a boundary layer term $\varepsilon P\left(x_{1}\right) \mathbf{w}(x / \varepsilon)$ dominates.

The result can be used for the development of the model-coupling strategies, 325 see [15] and 25].

\section{References}

[1] G. Allaire, Homogenization and two-scale convergence, SIAM J.Math.Anal., 23, 6 (1992), p. 1482-1518.

[2] G. Allaire, One-Phase Newtonian Flow, Chapter in [16, Homogenization and Porous Media, ed. U.Hornung, Springer, New-York, (1997), p. 45-68.

[3] G. Allaire, C. Conca, Boundary Layers in the Homogenization of a Spectral Problem in Fluid-Solid Structures, SIAM J.Math.Anal., Vol 29, No 2 (1997), p. $343-379$.

[4] W. Bangerth and R. Hartmann and G. Kanschat, deal.II - a General Purpose Object Oriented Finite Element Library, ACM Trans. Math. Softw., Vol. 33 (2007), p. 24/1-24/27.

[5] G. S. Beavers and D. D. Joseph, Boundary conditions at a naturally permeable wall, J. Fluid Mech., 30 (1967), p. 197-207.

[6] R. Becker, R. Rannacher, An optimal control approach to a posteriori error estimation in finite element methods, Acta Numerica, Vol. 10 (2001), p. $1-102$.

[7] F. Boyer, P. Fabrie, Mathematical Tools for the Study of the Incompressible Navier-Stokes Equations and Related Models, Applied Mathematical Sciences Vol. 183, Springer, New York, 2013. 
[8] F. Brezzi, M. Fortin, Mixed and hybrid finite element methods, SpringerVerlag New York, Inc., New York, NY, USA, 1991.

[9] T. Carraro, C. Goll, A. Marciniak-Czochra, A. Mikelić, Pressure jump interface law for the Stokes-Darcy coupling: Confirmation by direct numerical simulations, J. Fluid Mech., Vol. 732 (2013), p. 510-536.

[10] T. Carraro, C. Goll, A. Marciniak-Czochra, A. Mikelić, Effective interface conditions for the forced infiltration of a viscous fluid into a porous medium using homogenization, Comput. Methods Appl. Mech. Engrg., 292 (2015), p. $195-220$.

[11] C. Conca, F. Murat, O. Pironneau, The Stokes and Navier-Stokes equations with boundary conditions involving the pressure, Japanese journal of mathematics. New series, 20(2) (1994), p. 279-318.

[12] O. Coceal, S. E. Belchera, Canopy model of mean winds through urban areas, Q. J. R. Meteorol. Soc., 130 (2004), p. 13491372.

[13] P. Constantin, C. Foias, Navier-Stokes equations. University of Chicago Press, 1988.

[14] L. C. Evans, Partial Differential Equations: Second Edition (Graduate Studies in Mathematics), AMS, Providence, 2010.

[15] R. Helmig, B. Flemisch, M. Wolff, A. Ebigbo, H. Class, Model coupling for multiphase flow in porous media, Advances in Water Resources, 51 (2013), p. 5266 .

[16] U. Hornung, ed., Homogenization and Porous Media, Springer, New York, 1997.

[17] W. Jäger, A. Mikelić, On the Flow Conditions at the Boundary Between a Porous Medium and an Impervious Solid, in " Progress in Partial Differential Equations: the Metz Surveys 3 ", eds. M.Chipot, J.Saint Jean Paulin et 
I. Shafrir, $\pi$ Pitman Research Notes in Mathematics no. 314, p. 145-161, Longman Scientific and Technical, London, 1994.

[18] W. Jäger, A. Mikelić, On the boundary condition on the contact interface between a porous medium and a free fluid, Ann. Scuola Norm. Sup. Pisa Cl. Sci. (4), 23 (1996), p. 403-465.

[19] W. Jäger and A. Mikelić, On the interface boundary condition of Beavers, Joseph, and Saffman, SIAM J. Appl. Math., 60 (2000), p. 1111-1127.

[20] W. Jäger and A. Mikelić and N. Neuss, Asymptotic Analysis of the Laminar Viscous Flow Over a Porous Bed, SIAM Journal on Scientific Computing, Vol. 22(2001), p. 2006-2028.

[21] T. Levy, E. Sanchez-Palencia, On boundary conditions for fluid flow in porous media, International Journal of Engineering Science, 13 (1975), p. 923-940.

[22] R. Lipton, M. Avellaneda, Darcy's law for slow viscous flow past a trationary array of bubbles, Proc. Roy. Soc. Edinbourgh, 114A (1990), p. 71-79.

[23] A. Marciniak-Czochra, A. Mikelić, Effective pressure interface law for transport phenomena between an unconfined fluid and a porous medium using homogenization, SIAM Multiscale Model. Simul., Vol. 10, No. 2 (2012), p. 285-305.

[24] E. Marušić- Paloka, A. Mikelić, An Error Estimate for Correctors in the Homogenization of the Stokes and Navier-Stokes Equations in a Porous Medium, Boll. Unione Mat. Ital. , A(7) 10 (1996), no. 3, p. 661-671.

[25] K. Mosthaf, K. Baber, B. Flemisch, R. Helmig, A. Leijnse, I. Rybak, B. Wohlmuth, A coupling concept for two-phase compositional porous medium and single-phase compositional free flow. Water Resources Research, 47(10) (2011), p. 36 . 
[26] G. Nguetseng, A general convergence result for a functional related to the theory of homogenization. SIAM J. Math. Anal, 20:3 (1989), p. 608-623.

[27] E. Sanchez-Palencia, Non-homogeneous media and vibration theory, Springer lecture notes in physics 127, Springer-Verlag, Berlin, 1980.

[28] L. Tartar, Convergence of the homogenization process, Appendix of 27]

[29] C. Taylor, P. Hood, A numerical solution of the Navier-Stokes equations using the finite element technique. Computers and Fluids, 1(1) (1973), p. $73-100$. 